\title{
A hybrid semismooth quasi-Newton method for nonsmooth optimal control with PDEs
}

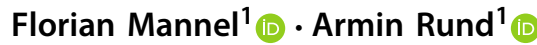

Received: 2 October 2019 / Revised: 16 March 2020 / Accepted: 9 June 2020 / Published online: 18 July 2020

(c) The Author(s) 2020

\begin{abstract}
We propose a semismooth Newton-type method for nonsmooth optimal control problems. Its particular feature is the combination of a quasi-Newton method with a semismooth Newton method. This reduces the computational costs in comparison to semismooth Newton methods while maintaining local superlinear convergence. The method applies to Hilbert space problems whose objective is the sum of a smooth function, a regularization term, and a nonsmooth convex function. In the theoretical part of this work we establish the local superlinear convergence of the method in an infinite-dimensional setting and discuss its application to sparse optimal control of the heat equation subject to box constraints. We verify that the assumptions for local superlinear convergence are satisfied in this application and we prove that convergence can take place in stronger norms than that of the Hilbert space if initial error and problem data permit. In the numerical part we provide a thorough study of the hybrid approach on two optimal control problems, including an engineering problem from magnetic resonance imaging that involves bilinear control of the Bloch equations. We use this problem to demonstrate that the new method is capable of solving nonconvex, nonsmooth large-scale real-world problems. Among others, the study addresses mesh independence, globalization techniques, and limited-memory methods. We observe throughout that algorithms based on the hybrid methodology are several times faster in runtime than their semismooth Newton counterparts.
\end{abstract}

Keywords Semismooth Newton methods · Quasi-Newton methods · Superlinear convergence $\cdot$ Nonsmooth optimal control $\cdot$ Bloch equations

Mathematics Subject Classification 49M05 - 49M15 • 49K20 • 49-04 • 90-04 • 90C06 • 90C30 - 90C53 - 90C56 - 90C90

Armin Rund

armin.rund@uni-graz.at

Florian Mannel

florian.mannel@uni-graz.at

1 University of Graz, Heinrichstr. 36, 8010 Graz, Austria 


\section{Introduction}

In Mannel and Rund (2019) we developed the convergence theory of a hybrid semismooth quasi-Newton method to solve structured operator equations in Banach spaces. In the present paper we address applications and numerical realizations of that work. Specifically, we propose a novel algorithm to solve infinite-dimensional nonsmooth optimization problems of the form

$$
\min _{u \in U} \hat{f}(u)+\frac{\gamma}{2}\|u\|_{U}^{2}+\varphi(u)
$$

show that its theoretical requirements are met by certain optimal control problems that are special instances of $(\mathrm{P})$, and provide an extensive numerical study for the new method on convex and nonconvex optimal control problems. In (P), $U$ is a Hilbert space, $\gamma>0, \varphi: U \rightarrow \mathbb{R} \cup\{+\infty\}$ is convex but possibly nonsmooth, and $\hat{f}: U \rightarrow \mathbb{R}$ is smooth but possibly nonconvex. The precise problem setting is given in Sect. 3. A prototypical example from PDE-constrained optimal control is

$$
\min _{u \in L^{2}(\Omega)} \frac{1}{2}\left\|y(u)-y_{d}\right\|_{L^{2}(\Omega)}^{2}+\frac{\alpha}{2}\|u\|_{L^{2}(\Omega)}^{2}+\beta\|u\|_{L^{1}(\Omega)} \quad \text { s.t. } \quad a \leq u \leq b \quad \text { a.e. in } \Omega,
$$

where $a<0<b$ and $\alpha, \beta>0$ are real numbers, $\Omega$ is a bounded Lipschitz domain, $y_{d} \in L^{2}(\Omega)$, and $y=y(u)$ is for $u \in L^{2}(\Omega)$ the solution of the semilinear equation $-\Delta y+y^{3}=u$ with appropriate boundary conditions. As $\hat{f}(u)=\frac{1}{2}\left\|y(u)-y_{d}\right\|_{L^{2}(\Omega)}^{2}$ in this instance of $(\mathrm{P})$, the evaluation of $\hat{f}$ and its derivatives requires PDE solves.

The new algorithm is a semismooth Newton-type method that exploits the presence of the smooth term $\nabla \hat{f}$ in the optimality conditions of (P) by applying a quasi-Newton method. Specifically, the operator $\nabla^{2} \hat{f}\left(u^{k}\right)$ that appears in semismooth Newton methods is replaced by a quasi-Newton approximation $B_{k}$. In PDE-constrained optimal control problems this lowers the runtime significantly because it omits the PDE solves that occur in the evaluation of Hessian-vector products $\nabla^{2} \hat{f}\left(u^{k}\right) d$ while maintaining superlinear convergence. Note that a direct application of quasi-Newton methods to semismooth equations cannot ensure superlinear convergence. For instance, Broyden's method on a piecewise affine (hence semismooth) equation in $\mathbb{R}$ may converge only r-linearly, cf. (Griewank 1987, Introduction). We present the hybrid method and its convergence properties for problems of the form $(\mathrm{P})$ in Sect. 3. Its application to a model problem from PDE-constrained optimal control, the time-dependent sparse optimal control of linear and semilinear heat equations subject to box constraints, constitutes Sect. 4 and concludes the theoretical part.

The remainder of the paper is devoted to numerics. We devise various numerical realizations of the hybrid method and compare them as part of an extensive numerical study. This study addresses many theoretical and practical aspects of the hybrid approach: We verify experimentally the superlinear convergence with respect to different norms, we investigate mesh independence properties, we compare different globalization strategies including a trust-region method, and we examine different quasi-Newton updates (Broyden, SR1, BFGS). 
The numerical study is based on two optimal control problems, starting with the time-dependent box-constrained sparse optimal control of the linear heat equation from the theoretical part. This model problem allows us to display very clearly the convergence properties of the hybrid method, e.g., its local superlinear convergence. The second problem deals with the design of radio-frequency pulses for magnetic resonance imaging, a topic from medical engineering. A realistic modeling yields a nonsmooth and nonconvex optimal control problem that serves as a benchmark for the performance of the hybrid approach on real-world applications. The numerical results underline that the hybrid approach can be competitive on such problems. Indeed, a previous version of the presented trust-region method formed the kernel of the code Rund et al. (2018a, b) that won the ISMRM challenge on radio-frequency pulse design in magnetic resonance imaging Grissom et al. (2017). Here we provide an improved successor.

Since quasi-Newton methods involve the Hilbert space structure of $U$ in an essential way, it may be surprising that the numerical results for both problems clearly indicate convergence of the control $u$ with respect to stronger norms than that of $U$. In Sect. 4 we establish rigorous theoretical results that explain this behavior for the control of the heat equation. It is related to the regularity of the problem and the quality of the initial approximation.

Although quasi-Newton methods have been applied to PDE-constrained optimal control problems, for instance in (Borzì and Schulz 2012, Chapter 4), Hinze and Kunisch (2001), and (Ulbrich 2011, Chapter 11), there are rather few infinitedimensional convergence results available for algorithms that incorporate quasiNewton methods and can handle nonsmoothness. We are aware of Sachs (1985), Griewank (1987), Muoi et al. (2013), Adly and Ngai (2018), but none of these yield superlinear convergence for $(\mathrm{P})$.

State-of-the-art methods for solving optimal control problems of the form $(\mathrm{P})$ are semismooth Newton methods, cf. Ito and Kunisch (2008), Hinze et al. (2009), Ulbrich (2011) and De los Reyes (2015). In particular, they have been applied successfully to sparse optimal control problems, cf., e.g., Stadler (2009), Amstutz and Laurain (2013), Herzog et al. (2012), Herzog et al. (2015), Kunisch et al. (2016) and Boulanger and Trautmann (2017). Since the problems that we address in the numerical study are of this type, we consistently compare the hybrid approach to semismooth Newton methods.

In finite-dimensional settings there are many works available that treat (modified and unmodified) quasi-Newton methods for nonsmooth equations. The idea to apply a quasi-Newton method to the smooth part of a structured nonsmooth equation, which is at the core of the hybrid approach, appears in Chen and Yamamoto (1992), Wang et al. (2011), Qi and Jiang (1997), Han and Sun (1997), Sun and Han (1997). Among these contributions, Han and Sun (1997) is the closest to our work because it is based on finding the root of a normal map; after reformulating the optimality conditions of (P) we are faced with the same task (which we tackle by the hybrid semismooth quasi-Newton method). However, Han and Sun (1997) does not address the infinitedimensional setting and, in finite dimensions, is less general than our approach in regard to the set of constraints, which in Han and Sun (1997) has to be polyhedral. On the other hand, Han and Sun (1997) offers a deeper treatment for the specific setting of normal maps with polyhedral sets in finite dimensions. 
This paper is organized as follows. Section 2 specifies some notions that are important for this work, e.g., semismoothness. In Sect. 3 we provide the problem under consideration in full detail, introduce the hybrid method, and establish convergence results for it. Section 4 discusses the application of the method to sparse optimal control of linear and semilinear heat equation with box constraints. In Sect. 5 we comment on implementation issues. Section 6 contains the numerical study and in Sect. 7 we draw conclusions from this work. In "Appendix A" we provide the algorithm that we found most effective in the numerical studies - a matrix-free limited-memory truncated trust-region variant of the hybrid method.

\section{Preliminaries}

In this short section we fix the notation, recall the notion of proximal maps, and specify which concept of semismoothness we use.

We set $\mathbb{N}:=\{1,2,3, \ldots\}$. All linear spaces are real linear spaces. Let $X$ and $Y$ be Banach spaces. We denote $\mathbb{B}_{\delta}(\bar{x}):=\left\{x \in X:\|x-\bar{x}\|_{X}<\delta\right\}$ for $\delta>0$ and $\bar{x} \in X$. Moreover, $\mathcal{L}(X, Y)$ represents the space of bounded linear maps from $X$ to $Y$. If $X$ can be continuously embedded into $Y$, this is indicated by $X \hookrightarrow Y$. In the Hilbert space $U$ we write $(v, w)_{U}$ for the scalar product of $v, w \in U$ and $(v, \cdot)_{U}$ for the linear operator $w \mapsto(v, w)_{U}$ from $U$ to $\mathbb{R}$. Furthermore, we recall the definition and elementary properties of the proximal map.

Definition 1 Let $U$ be a Hilbert space and let $\gamma>0$. Let $\varphi: U \rightarrow \mathbb{R} \cup\{+\infty\}$ be a proper, convex, and lower semicontinuous function and denote its effective domain by $C:=\{u \in U: \varphi(u)<+\infty\}$. The proximal mapping of $\varphi_{\gamma}:=\frac{\varphi}{\gamma}$ is given by

$$
\operatorname{Prox}_{\varphi_{\gamma}}: U \rightarrow U, \quad \operatorname{Prox}_{\varphi_{\gamma}}(u):=\operatorname{argmin}_{\tilde{u} \in C}\left[\frac{1}{2}\|\tilde{u}-u\|_{U}^{2}+\varphi_{\gamma}(\tilde{u})\right]
$$

It is easy to see that $\operatorname{Prox}_{\varphi_{\gamma}}$ is single-valued, has image $C$, and satisfies the relation

$$
\hat{u}=\operatorname{Prox}_{\varphi_{\gamma}}(u) \quad \Longleftrightarrow \quad \gamma(u-\hat{u}) \in \partial \varphi(\hat{u})
$$

for $u, \hat{u} \in U$ and arbitrary $\gamma>0$, where $\partial \varphi$ denotes the convex subdifferential of $\varphi$. If $\varphi$ is the characteristic function of a closed convex set, then $\operatorname{Prox}_{\varphi_{\gamma}}$ is the projection onto that set. More on proximal mappings in Hilbert spaces can be found in (Bauschke and Combettes 2017, Section 24), for instance.

We will use the rather general notion of semismoothness from (Ulbrich 2011, Definition 3.1) that includes, for instance, Newton differentiability, cf. (Ito and Kunisch 2008, Definition 8.10).

Definition 2 Let $X, Y$ be Banach spaces and let $\bar{x} \in X$. Let $G: X \rightarrow Y$ be continuous in an open neighborhood of $\bar{x}$. Moreover, let $\partial G: X \rightrightarrows \mathcal{L}(X, Y)$ satisfy $\partial G(x) \neq \emptyset$ for all $x \in X$. We say that $G$ is semismooth at $\bar{x}$ with respect to $\partial G$ iff there holds

$$
\sup _{M \in \partial G(\bar{x}+h)}\|G(\bar{x}+h)-G(\bar{x})-M h\|_{Y}=o\left(\|h\|_{X}\right) \quad \text { for }\|h\|_{X} \rightarrow 0 .
$$


The set-valued mapping $\partial G: X \rightrightarrows \mathcal{L}(X, Y)$ is called a generalized derivative of $G$. For $x \in X$ every $M \in \partial G(x)$ is called a generalized differential of $G$ at $x$.

\section{Problem setting, algorithm, and convergence results}

In this section we introduce the problem class in full detail and present the hybrid method to solve it. We provide its convergence properties and recall a result concerning local uniform invertibility of generalized differentials.

\subsection{Problem setting and algorithm}

Throughout this work we consider optimization problems of the form

$$
\min _{u \in U} \underbrace{\hat{f}(u)+\frac{\gamma}{2}\|u\|_{U}^{2}}_{=: f(u)}+\varphi(u)
$$

the details of which are contained in the following assumption.

Assumption 1 Let the following conditions be satisfied.

1) $U$ is a Hilbert space.

2) (P) has a local solution, denoted $\bar{u} \in U$.

3) The function $\varphi: U \rightarrow \mathbb{R} \cup\{+\infty\}$ is proper, convex, and lower semicontinuous.

4) The function $\hat{f}: U \rightarrow \mathbb{R}$ is continuously differentiable.

5) There is a Banach space $Q \hookrightarrow U$ such that

$$
\nabla \hat{f}(u) \in Q \text { for all } u \in U
$$

such that

$$
\nabla \hat{f}: U \rightarrow Q \text { is differentiable }
$$

and such that

$$
\nabla^{2} \hat{f}: U \rightarrow \mathcal{L}(U, Q) \text { is locally Hölder continuous. }
$$

6) There are $\gamma, \delta, C_{M}>0$ such that

$$
\operatorname{Prox}_{\varphi_{\gamma}}: Q \rightarrow U \text { is semismooth at } \bar{q}:=-\frac{1}{\gamma} \nabla \hat{f}(\bar{u})
$$

and such that for each $q \in \mathbb{B}_{\delta}(\bar{q})$ and all $M \in \partial \operatorname{Prox}_{\varphi_{\gamma}}(q)$ there holds

$$
\|M\|_{\mathcal{L}(Q, U)} \leq C_{M}
$$


7) Defining

$$
H: Q \rightarrow Q, \quad H(q):=\nabla \hat{f}\left(\operatorname{Prox}_{\varphi_{\gamma}}(q)\right)+\gamma q
$$

with generalized derivative

$$
\partial H: Q \rightrightarrows \mathcal{L}(Q, Q), \quad \partial H(q):=\left\{\nabla^{2} \hat{f}(\bar{u}) \circ M+\gamma I: M \in \partial \operatorname{Prox}_{\varphi_{\gamma}}(q)\right\}
$$

there are $\bar{\delta}, C_{\bar{M}^{-1}}>0$ such that for each $q \in \mathbb{B}_{\bar{\delta}}(\bar{q})$ all $\bar{M} \in \partial H(q)$ are invertible and satisfy

$$
\left\|\bar{M}^{-1}\right\|_{\mathcal{L}(Q, Q)} \leq C_{\bar{M}^{-1}} .
$$

Remark 1 Note that $\operatorname{Prox}_{\varphi_{\gamma}}$ is an operator from $U$ to $U$, but is required to be semismooth from $Q$ to $U$ in 6). Note, furthermore, that $\bar{q} \in Q$ holds in 6) due to (2).

Remark 2 Under Assumption 1 there are constants $L_{P}, L_{\nabla}>0$ such that

$$
\left\|\operatorname{Prox}_{\varphi_{\gamma}}(q)-\operatorname{Prox}_{\varphi_{\gamma}}(\bar{q})\right\|_{U} \leq L_{P}\|q-\bar{q}\|_{Q} \quad \text { and } \quad\|\nabla \hat{f}(u)-\nabla \hat{f}(\bar{u})\|_{Q} \leq L_{\nabla}\|u-\bar{u}\|_{U}
$$

are satisfied for all $q$ close to $\bar{q}$, respectively, for all $u$ close to $\bar{u}$. The constants $L_{P}$ and $L_{\nabla}$ will appear in the convergence results below.

Remark 3 It would be enough to require 3)-5) only locally around $\bar{u}$.

Since $f$ can be nonconvex and since $\varphi$ can be nonsmooth, $(\mathrm{P})$ is a nonconvex and nonsmooth optimization problem, in general. It may also feature a convex admissible set, as $\varphi$ is extended real-valued. We tackle $(\mathrm{P})$ by reformulating its first order optimality condition as operator equation $H(q)=0$. The approach to use Robinson's normal map Robinson (1992) for the reformulation is inspired by (Pieper 2015, Section 3), which is one of the rather few references that we are aware of where a prox-based reformulation of the optimality conditions is used in the context of infinite dimensional PDE-constrained optimal control. This approach is, however, quite common in finite dimensional optimization, in particular in connection with first order methods, cf., e.g., Beck (2017) and Parikh and Boyd (2014). Also, let us point out that semismoothness of proximal maps is addressed in (Xiao et al. 2018, Section 3) and (Milzarek 2016, Section 3.3) for finite dimensions as well as in (Pieper 2015, Section 3.3) for infinite dimensions.

Lemma 1 Let Assumption 1 hold. Then $\bar{u}$ satisfies the necessary optimality condition $0 \in \nabla f(\bar{u})+\partial \varphi(\bar{u})$ of $(\mathrm{P})$ and $\bar{q}$ satisfies $H(\bar{q})=0$, where $H$ is given by (3). Moreover, for any $\hat{q} \in Q$ with $H(\hat{q})=0$ the point $\hat{u}:=\operatorname{Prox}_{\varphi_{\gamma}}(\hat{q})$ satisfies $0 \in \nabla f(\hat{u})+\partial \varphi(\hat{u})$.

If the objective in $(\mathrm{P})$ is convex, then any such $\hat{u}$ is a global solution of $(\mathrm{P})$.

Proof It is well-known that the local solution $\bar{u}$ of (P) satisfies $0 \in \nabla f(\bar{u})+\partial \varphi(\bar{u})$. Since $\bar{q}=-\frac{1}{\gamma} \nabla \hat{f}(\bar{u})$ by definition, we obtain $\gamma(\bar{q}-\bar{u})=-\nabla f(\bar{u}) \in \partial \varphi(\bar{u})$, hence $\bar{u}=\operatorname{Prox}_{\varphi_{\gamma}}(\bar{q})$ by (1). Inserting this into $\bar{q}=-\frac{1}{\gamma} \nabla \hat{f}(\bar{u})$ implies $H(\bar{q})=0$.

If $\hat{q} \in Q$ with $H(\hat{q})=0$ is given and we set $\hat{u}:=\operatorname{Prox}_{\varphi_{\gamma}}(\hat{q}) \in U$, then we have

$$
H(\hat{q})=0 \quad \Longrightarrow \quad-\nabla \hat{f}(\hat{u})-\gamma \hat{u}=\gamma(\hat{q}-\hat{u}) \quad \Longrightarrow \quad-\nabla f(\hat{u}) \in \partial \varphi(\hat{u}),
$$


where the final implication involves (1). The last identity yields $0 \in \nabla f(\hat{u})+\partial \varphi(\hat{u})$.

The assertion concerning convexity is true since it just restates that the necessary optimality condition is sufficient for global optimality in convex optimization.

Next we provide the new algorithm. It aims at solving the operator equation $H(q)=$ 0 . Note that $H$ acts on the artificial variable $q$ that is related to the control $u$ by $u^{k}=\operatorname{Prox}_{\varphi_{\gamma}}\left(q^{k}\right)$ for $k \geq 1$, respectively, $\bar{q}=-\frac{1}{\gamma} \nabla \hat{f}(\bar{u})$.

The key idea of the new method is to replace the Hessian $\nabla^{2} \hat{f}\left(u^{k}\right)$ that appears in semismooth Newton methods by a quasi-Newton approximation $B_{k}$. In contrast, the generalized derivative of the proximal map $\operatorname{Prox}_{\varphi_{\gamma}}$ is left unchanged. The algorithm thus combines a quasi-Newton method with a semismooth Newton method and can be regarded as a hybrid approach. It reads as follows.

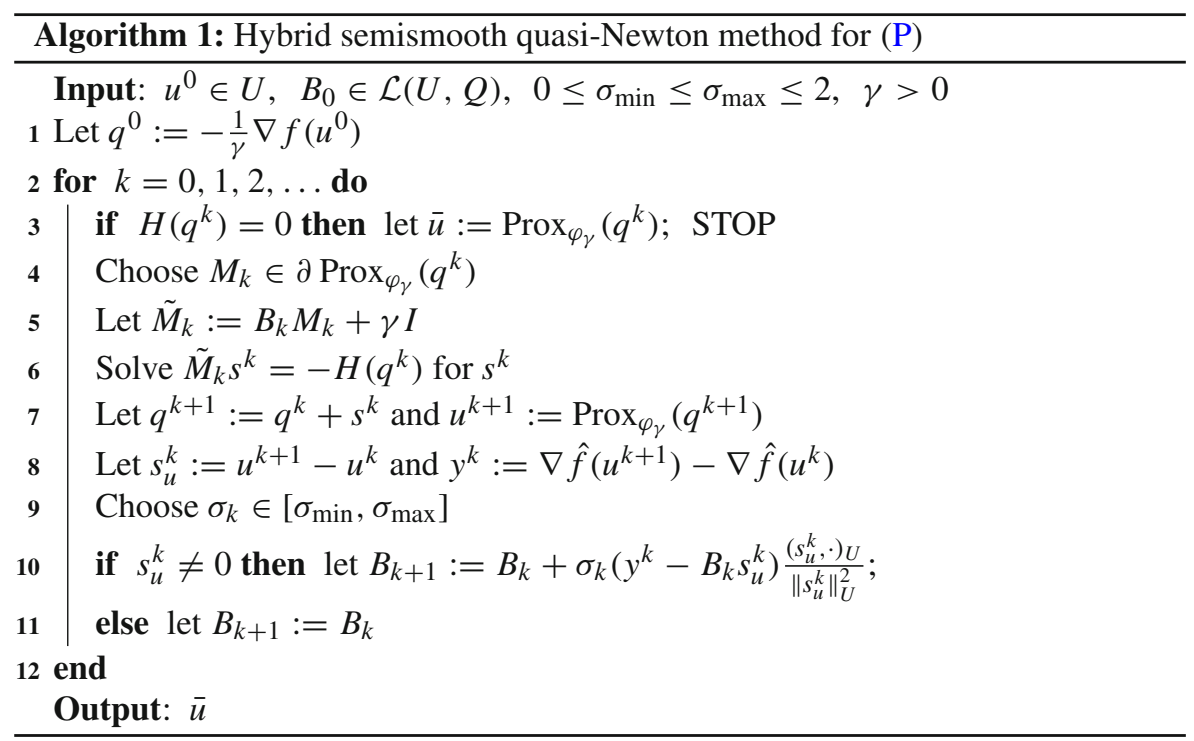

In the numerical study in Sect. 6 we work exclusively with $\left(\sigma_{k}\right) \equiv 1$ in line 9 , i.e., the classical Broyden update, as already this simple choice results in efficient algorithms. Also, we will compare this update to other update formulas, specifically

$$
B_{k+1}=B_{k}+\left(y^{k}-B_{k} s_{u}^{k}\right) \frac{\left(y^{k}-B_{k} s_{u}^{k}, \cdot\right)_{U}}{\left(y^{k}-B_{k} s_{u}^{k}, s_{u}^{k}\right)_{U}}
$$

and

$$
B_{k+1}=B_{k}+y^{k} \frac{\left(y^{k}, \cdot\right)_{U}}{\left(y^{k}, s_{u}^{k}\right)_{U}}-B_{k} s_{u}^{k} \frac{\left(B_{k} s_{u}^{k}, \cdot\right)_{U}}{\left(B_{k} s_{u}^{k}, s_{u}^{k}\right)_{U}} .
$$

Moreover, it should be clear that Algorithm 1 needs to be globalized for the numerical experiments. We will observe in the first half of Sect. 6 that if $\hat{f}$ is convex, then a simple line search suffices for this. In contrast, if $(\mathrm{P})$ is severely nonlinear, then a 
trust-region globalization yields better results, cf. the optimal control of the Bloch equation in the second half of Sect. 6.

\subsection{Convergence results}

For the iterates $\left(q^{k}\right)$ of Algorithm 1 we have the following convergence result.

Theorem 1 Let Assumption 1 hold and let $\mu \in(0,1)$. Then:

1) There exists $\varepsilon>0$ such that for every initial pair $\left(u^{0}, B_{0}\right) \in U \times \mathcal{L}(U, Q)$ with $\left\|u^{0}-\bar{u}\right\|_{U}<\varepsilon$ and $\left\|B_{0}-\nabla^{2} \hat{f}\left(u^{0}\right)\right\|_{\mathcal{L}(U, Q)}<\varepsilon$, Algorithm 1 either terminates after finitely many iterations or generates a sequence of iterates $\left(q^{k}\right)$ that converges $q$-linearly with rate $\mu$ to $\bar{q}$. If, in addition, $\sigma_{\min }, \sigma_{\max } \in(0,2)$ in Algorithm 1 and $B_{0}-\nabla^{2} \hat{f}(\bar{u})$ is compact, then the convergence is $q$-superlinear.

2) If $\nabla^{2} \hat{f}\left(u^{0}\right)-\nabla^{2} \hat{f}(\bar{u})$ is compact, then the compactness of $B_{0}-\nabla^{2} \hat{f}(\bar{u})$ in 1$)$ can be replaced by the compactness of $B_{0}-\nabla^{2} \hat{f}\left(u^{0}\right)$.

Proof This follows from (Mannel and Rund 2019, Theorem 4.2 and Theorem 4.18) for $F(u):=\nabla \hat{f}(u), G(q):=\operatorname{Prox}_{\varphi_{\gamma}}(q), \hat{G}(q):=\gamma q$, and $V:=Q$. We remark that the results in Mannel and Rund (2019) require $\left(q^{0}, B_{0}\right)$ to be close to $\left(\bar{q}, \nabla^{2} \hat{f}(\bar{u})\right)$, but this is implied by the fact that $\left(u^{0}, B_{0}\right)$ is close to $\left(\bar{u}, \nabla^{2} \hat{f}(\bar{u})\right)$ since $\left\|q^{0}-\bar{q}\right\|_{Q}=$ $\left\|\frac{1}{\gamma} \nabla \hat{f}\left(u^{0}\right)-\frac{1}{\gamma} \nabla \hat{f}(\bar{u})\right\|_{Q} \leq \frac{L_{\nabla}}{\gamma}\left\|u^{0}-\bar{u}\right\|_{U}$.

Regarding convergence of $\left(u^{k}\right),\left(\nabla \hat{f}\left(u^{k}\right)\right)$ and $\left(H\left(q^{k}\right)\right)$ we obtain the following.

Corollary 1 Let Assumption 1 hold and let $\left(q^{k}\right)$ be generated by Algorithm 1. If $\left(q^{k}\right)$ converges $q$-linearly ( $q$-superlinearly) to $\bar{q}$, then:

1) $\left(u^{k}\right)$ converges $r$-linearly ( $r$-superlinearly) to $\bar{u}$ and satisfies, for all $k$ sufficiently large, $\left\|u^{k}-\bar{u}\right\|_{U} \leq L_{P}\left\|q^{k}-\bar{q}\right\|_{Q}$.

2) $\left(\nabla \hat{f}\left(u^{k}\right)\right)$ converges $r$-linearly (r-superlinearly) to $\nabla \hat{f}(\bar{u})$ and satisfies, for all $k$ sufficiently large, $\left\|\nabla \hat{f}\left(u^{k}\right)-\nabla \hat{f}(\bar{u})\right\|_{Q} \leq L_{\nabla}\left\|u^{k}-\bar{u}\right\|_{U}$ and $\| \nabla \hat{f}\left(u^{k}\right)-$ $\nabla \hat{f}(\bar{u})\left\|_{Q} \leq L_{\nabla} L_{P}\right\| q^{k}-\bar{q} \|_{Q}$.

3) $\left(H\left(q^{k}\right)\right)$ converges $r$-linearly (q-superlinearly) to zero and satisfies, for all $k$ sufficiently large, $\left\|H\left(q^{k}\right)\right\|_{Q} \leq\left(L_{\nabla} L_{P}+\gamma\right)\left\|q^{k}-\bar{q}\right\|_{Q}$.

Proof This follows from (Mannel and Rund 2019, Corollary 4.4 and Corollary 4.20) for $F(u):=\nabla \hat{f}(u), G(q):=\operatorname{Prox}_{\varphi_{\gamma}}(q), \hat{G}(q):=\gamma q$, and $V:=Q$.

\subsection{A general approach for local uniform invertibility}

The following result is taken from (Pieper 2015, Section 3). It allows to conveniently establish condition 7) of Assumption 1.

Lemma 2 Let conditions 1)-6) of Assumption 1 hold and let $H$ and $\partial H$ be given by (3), respectively, (4). Moreover, suppose that $\partial \operatorname{Prox}_{\varphi_{\gamma}}$ can be extended to $U$ in such a way that $\partial \operatorname{Prox}_{\varphi_{\gamma}}(u) \subset \mathcal{L}(U, U)$ for every $u \in U$. Then condition 7) of Assumption 1 is satisfied if there exist $v, \hat{\delta}>0$ such that for each $u \in \mathbb{B}_{\hat{\delta}}(\bar{u})$ and all $M \in \partial \operatorname{Prox}_{\varphi_{\gamma}}(u)$ we have 
$-\|M\|_{\mathcal{L}(U, U)} \leq 1$

- $(M h, h)_{U} \geq 0$ for all $h \in U$,

- $\left(M h_{1}, h_{2}\right)_{U}=\left(h_{1}, M h_{2}\right)_{U}$ for all $h_{1}, h_{2} \in U$,

- and

$$
\gamma(h, M h)_{U}+\left(\nabla^{2} \hat{f}(\bar{u}) M h, M h\right)_{U} \geq v(h, M h)_{U} \quad \text { for all } h \in U .
$$

Proof This is (Pieper 2015, Lemma 3.15) for the situation at hand.

Remark 4 Inequality (5) is, in particular, valid if $\nabla^{2} \hat{f}(\bar{u})$ is positive semidefinite.

\section{Application to PDE-constrained optimal control}

In this section we show for a model problem how the hybrid approach can be applied to PDE-constrained optimal control problems. These problems are well-suited for the application of the hybrid method because the assumptions for fast local convergence are typically satisfied.

\subsection{An important proximal map}

To facilitate the discussion of the model problem in Sect. 4.2, we study the associated proximal map in this section. To this end, let $N \in \mathbb{N}, T>0$, and $U:=L^{2}(I)^{N}$, where $I:=(0, T)$ for some $T>0$. We are interested in the proximal map of

$$
\varphi: U \rightarrow \mathbb{R} \cup\{+\infty\}, \quad \varphi(u):=\delta_{U_{\mathrm{ad}}}(u)+\sum_{i=1}^{N} \beta_{i}\left\|u_{i}\right\|_{L^{1}(I)},
$$

where $\beta_{i}, 1 \leq i \leq N$, are nonnegative real numbers, $U_{\mathrm{ad}}$ is given by

$$
U_{\mathrm{ad}}:=\left\{u=\left(u_{1}, \ldots, u_{N}\right)^{T} \in U: a_{i} \leq u_{i} \leq b_{i} \text { a.e. in } I, 1 \leq i \leq N\right\}
$$

for functions $a, b \in L^{\infty}(I)^{N}$ that satisfy $a \leq b$ a.e. in $I$ (the inequality is meant componentwise), and $\delta_{U_{\mathrm{ad}}}: U \rightarrow\{0,+\infty\}$ denotes the characteristic function of $U_{\text {ad. }}$. For reasons that become clear in Sect. 4.2, we fix positive weights $\alpha_{1}, \ldots, \alpha_{N}$ and endow $U$ with the norm $\|u\|_{U}:=\left(\sum_{i=1}^{N} \alpha_{i}\left\|u_{i}\right\|_{L^{2}(I)}^{2}\right)^{1 / 2}$. This norm is equivalent to the standard norm in $U$ and it is derived from a scalar product, hence $U$ is a Hilbert space with respect to it. We write $\Pi_{U_{\mathrm{ad}}}: U \rightarrow U$ for the projection onto $U_{\text {ad }}$, i.e.,

$$
\Pi_{U_{\mathrm{ad}}}(u):=\max (a, \min (u, b)) \text { for a.e. } x \in I .
$$


Furthermore, let us introduce the soft-shrinkage operator $\sigma: U \rightarrow U$, which is given componentwise for $1 \leq i \leq N$ and the constants $\alpha_{i}>0$ and $\beta_{i} \geq 0$ by

$\sigma_{i}(u)(t):=\rho_{i}\left(u_{i}(t)\right) \quad$ for $\quad \rho_{i}: \mathbb{R} \rightarrow \mathbb{R}, \quad \rho_{i}(s):=\left(s-\frac{\beta_{i}}{\alpha_{i}}\right)^{+}+\left(s+\frac{\beta_{i}}{\alpha_{i}}\right)^{-}$

where $(r)^{+}:=\max (0, r)$ and $(r)^{-}:=\min (0, r)$ for all $r \in \mathbb{R}$.

The proximal map for $\gamma=1$ can now be characterized as follows.

Lemma 3 The proximal map $\operatorname{Prox}_{\varphi_{1}}: U \rightarrow U_{\mathrm{ad}}$ is given by $\operatorname{Prox}_{\varphi_{1}}=\Pi_{U_{\mathrm{ad}}} \circ \sigma$.

Proof This can be established as in (Pieper 2015, Section 3.3) or through direct computation.

It is important to note that $\operatorname{Prox}_{\varphi_{1}}$ is semismooth.

Lemma $4 \operatorname{Prox}_{\varphi_{1}}$ is semismooth at every $q \in Q$ when considered as a mapping from $Q:=C([0, T])^{N}$ to $U$ with respect to the generalized derivative $\partial \operatorname{Prox}_{\varphi_{1}}(q) \subset$ $\mathcal{L}(Q, U)$ given by

$$
\partial \operatorname{Prox}_{\varphi_{1}}(q):=\bigcup_{\substack{r \in L^{\infty}(I)^{N} \text { with } \\ 0 \leq r \leq 1 \text { a.e. in } I}}\{M(q, r)\},
$$

where $0 \leq r \leq 1$ is meant componentwise and $M=M(q, r) \in \mathcal{L}(Q, U)$ is for $(q, r) \in Q \times L^{\infty}(I)^{N}$ defined as

$$
(M h)_{i}(t):= \begin{cases}h_{i}(t) & \text { if }\left|q_{i}(t)\right|>\frac{\beta_{i}}{\alpha_{i}} \wedge \sigma_{i}(q)(t) \in\left(a_{i}(t), b_{i}(t)\right), \\ 0 & \text { if }\left|q_{i}(t)\right|<\frac{\beta_{i}}{\alpha_{i}} \vee \sigma_{i}(q)(t) \notin\left[a_{i}(t), b_{i}(t)\right] \\ r_{i}(t) h_{i}(t) & \text { else }\end{cases}
$$

if $i \in\{1, \ldots, N\}$ is such that $\beta_{i}>0$. If $\beta_{i}=0$, then the conditions involving $\frac{\beta_{i}}{\alpha_{i}}$ have to be dropped in (6) and there holds $\sigma_{i}(q)=q_{i}$. In any case, $\|M\|_{\mathcal{L}(Q, U)} \stackrel{\alpha_{i}}{\leq}$ $T^{\frac{1}{2}}\left(\sum_{i=1}^{N} \alpha_{i}\right)^{\frac{1}{2}}$ holds for each $q \in Q$ and all $M \in \partial \operatorname{Prox}_{\varphi_{1}}(q)$.

Proof Since $\operatorname{Prox}_{\varphi_{1}}=\Pi_{U_{\mathrm{ad}}} \circ \sigma$, respectively, $\operatorname{Prox}_{\varphi_{1}}=\Pi_{U_{\mathrm{ad}}}$ is a superposition operator, the representation (6) can, for instance, be deduced from (Hinze et al. 2009, Theorem 2.13). The estimate for $\|M\|_{\mathcal{L}(Q, U)}$ follows since for $h \in Q$ with $\|h\|_{Q} \leq 1$ we have $(M h)_{i}(t) \leq 1$ for a.e. $t \in I$ and all $1 \leq i \leq N$.

Remark 5 We emphasize that the superposition operator $\Pi_{U_{\mathrm{ad}}} \circ \sigma$ is not semismooth from $U$ to $U$, cf. Ulbrich (2011); Schiela (2008). Correspondingly, the role of the additional space $Q$ in Assumption 1 is to ensure the necessary norm gap. In turn, the demand for $\nabla \hat{f}$ to map $U$ to $Q$, cf. 5) of Assumption 1, is a smoothing property. 


\subsection{Time-dependent control of the heat equation}

\subsubsection{The linear heat equation}

Let $N \in \mathbb{N}, Q:=C([0, T])^{N}, U:=L^{2}(I)^{N}$, and $Y:=P:=W\left(I ; L^{2}(\Omega), H_{0}^{1}(\Omega)\right)$, where $W\left(I ; L^{2}(\Omega), H_{0}^{1}(\Omega)\right)$ is the usual solution space for weak solutions of the heat equation, cf., e.g., (Hinze et al. 2009, (1.53)) or (Chipot 2000, (11.12)). We consider the optimal tracking of the linear heat equation in $I \times \Omega$ with $N$ time-dependent controls $u(t)=\left(u_{1}(t), \ldots, u_{N}(t)\right)^{T}$, where $\Omega \subset \mathbb{R}^{d}, 1 \leq d \leq 3$, is a nonempty and bounded Lipschitz domain, and the time domain is $I:=(0, T)$ for a fixed final time $T>0$ :

$$
\begin{gathered}
\min _{(y, u) \in Y \times U_{\mathrm{ad}}} \frac{1}{2}\left\|y-y_{\mathrm{d}}\right\|_{L^{2}\left(I \times \Omega_{\mathrm{obs}}\right)}^{2}+\sum_{i=1}^{N} \frac{\alpha_{i}}{2}\left\|u_{i}\right\|_{L^{2}(I)}^{2}+\sum_{i=1}^{N} \beta_{i}\left\|u_{i}\right\|_{L^{1}(I)} \\
\text { s.t. } \begin{cases}y_{t}-\Delta y=\sum_{i=1}^{N} g_{i}(x) u_{i}(t) & \text { in } I \times \Omega, \\
y=0 & \text { on } \Sigma, \\
y(0, x)=y_{0}(x) & \text { in } \Omega,\end{cases}
\end{gathered}
$$

where $\Sigma:=I \times \partial \Omega$. Moreover, $y_{\mathrm{d}} \in L^{2}\left(I \times \Omega_{\text {obs }}\right)$ is the desired state, $\Omega_{\text {obs }} \subset \Omega$ is the observation domain, $\alpha_{i}>0$ are the control cost parameters per control function, $\beta_{i} \geq 0$ influences the size of the support of $u_{i}, y_{0} \in L^{2}(\Omega)$ is the initial state, and $g_{i} \in L^{2}(\Omega)$ are fixed spatial functions whose support is denoted by $\omega_{i} \subset \Omega$, $1 \leq i \leq N$. For instance, $g_{i}$ could be the characteristic function $\chi_{\omega_{i}}$ of a given control domain $\omega_{i} \subset \Omega$. The set of admissible controls is given by

$$
U_{\mathrm{ad}}:=\left\{u=\left(u_{1}, \ldots, u_{N}\right)^{T} \in U: a_{i} \leq u_{i} \leq b_{i} \text { a.e. in } I, 1 \leq i \leq N\right\}
$$

with functions $a, b \in L^{\infty}(I)^{N}$ that satisfy $a \leq b$ a.e. in $I$. By using the same norm on $U$ as in Sect. 4.1 we can regard (OCP) as a special instance of (P) with $\gamma=1$. From (Tröltzsch 2010, Theorem 3.13) we obtain that for every $u \in U$ there exists a unique $y=y(u) \in Y$ such that the PDE-constraints in (OCP) are satisfied; the dependence $u \mapsto y(u)$ is linear and continuous from $U$ to $Y$. This implies that the solution operator $u \mapsto y(u)$ is infinitely many times continuously differentiable. Thus, $\hat{f}(u):=\frac{1}{2}\left\|y(u)-y_{\mathrm{d}}\right\|_{L^{2}\left(I \times \Omega_{\mathrm{obs}}\right)}^{2}$ is continuously differentiable from $U$ to $\mathbb{R}$. Since the control reduced version $(\mathrm{P})$ of $(\mathrm{OCP})$ is a convex problem with strongly convex objective, it is standard to show that it possesses a unique solution $\bar{u} \in U_{\text {ad }}$; the associated state is denoted by $\bar{y}:=y(\bar{u}) \in Y$. We can now derive the following result.

Lemma 5 For the control reduced version of (OCP) the mapping $H$ defined in (3) is for $\gamma=1$ given by

$H: Q \rightarrow Q, \quad H_{i}(q)(t)=\int_{\omega_{i}} g_{i}(x) p\left(\Pi_{U_{\mathrm{ad}}}(\sigma(q))\right)(t, x) d x+\alpha_{i} q_{i}(t), \quad 1 \leq i \leq N$ 
Here, $p=p(u) \in P$ is the adjoint state, i.e., the unique solution of the adjoint equation

$$
\begin{cases}-p_{t}-\Delta p=\chi_{I \times \Omega_{\mathrm{obs}}} \cdot\left(y(u)-y_{d}\right) & \text { in } I \times \Omega \\ p=0 & \text { on } \Sigma, \\ p(T)=0 & \text { in } \Omega\end{cases}
$$

Proof Adjoint calculus yields $(\nabla \hat{f}(u))_{i}(t)=\int_{\omega_{i}} g_{i}(x) p(t, x) / \alpha_{i} \mathrm{~d} x$ for $1 \leq i \leq N$, where $p=p(u)$ is the adjoint state. Inserting this in $H(q)=\nabla \hat{f}\left(\operatorname{Prox}_{\varphi_{1}}(q)\right)+q$ yields (8) due to Lemma 3. Moreover, the assumptions on the problem data imply $p(u) \in P$ for the solution of the adjoint equation, cf. (Tröltzsch 2010, Lemma 3.17). To show that $H$ maps to $Q$ we deduce from the continuous embedding $P \hookrightarrow$ $C\left([0, T] ; L^{2}(\Omega)\right)$, cf. (Chipot 2000, Theorem 11.4), that $p(u) \in C\left([0, T] ; L^{2}(\Omega)\right)$. Therefore, defining $\lambda=\lambda(u)$ by $\lambda_{i}(t):=\int_{\omega_{i}} g_{i}(x) p(t, x) / \alpha_{i} \mathrm{~d} x, t \in[0, T]$, where $1 \leq i \leq N$, implies $\lambda \in Q$. Since $\lambda(u)=\nabla \hat{f}(u)$, it follows that $\nabla \hat{f}$ maps $U$ to $Q$ and hence that $H$ maps to $Q$.

Remark 6 The proof of Lemma 5 demonstrates that we have to choose $Q$ in such a way that $t \mapsto \int_{\omega_{i}} g_{i}(x) p(t, x) \mathrm{d} x$ belongs to $Q$, where $p$ solves (9). Thus, the available regularity of the adjoint state $p$, respectively, of the multiplier $\lambda$, restricts the choice of $Q$. If additional regularity is available, then $Q$ may be chosen as a space of smoother functions than $C([0, T])^{N}$. For instance, from (Hinze et al. 2009, Theorem 1.39) we deduce that if $\Omega_{\mathrm{obs}}=\Omega$ and $y_{d} \in Y$, then there holds $\frac{\partial p(u)}{\partial t} \in$ $W\left(I ; L^{2}(\Omega), H_{0}^{1}(\Omega)\right)$, hence $p(u) \in H^{1}\left(I ; H_{0}^{1}(\Omega)\right)$. This implies $\lambda \in Q$ for the choice $Q:=H^{1}(I)^{N}$. In fact, using $W\left(I ; L^{2}(\Omega), H_{0}^{1}(\Omega)\right) \hookrightarrow C\left([0, T] ; L^{2}(\Omega)\right)$ we obtain $p \in C^{1}\left([0, T] ; L^{2}(\Omega)\right)$, which implies $\lambda \in Q$ for $Q:=C^{1}([0, T])^{N}$. We stress that Lemma 5 is valid for all these choices of $Q$.

Assumption 1 holds unconditionally for (OCP).

Lemma 6 The control reduced version of (OCP) fulfills Assumption 1 with $H: Q \rightarrow$ $Q$ given by (8). Moreover, $H$ has a unique root $\bar{q} \in Q$.

Proof Assumption 1 holds Conditions 1)-4) of Assumption 1 were already established, cf. the remarks above Lemma 5. In the proof of Lemma 5 we have demonstrated that $\nabla \hat{f}$ maps $U$ to $Q$. Since $\nabla \hat{f}: U \rightarrow Q$ is linear and continuous, it is infinitely many times continuously differentiable. This yields 5). Condition 6) follows from Lemma 4. To establish 7) we use Lemma 2. The representation in Lemma 4 shows that $\partial \operatorname{Prox}_{\varphi_{1}}(q), q \in Q$, can be extended in a canonical way to $\partial \operatorname{Prox}_{\varphi_{1}}(u), u \in U$. From the linearity of $u \mapsto y(u)$ we obtain that $\hat{f}(u)=\frac{1}{2}\left\|y(u)-y_{d}\right\|_{L^{2}\left(I \times \Omega_{\mathrm{obs}}\right)}^{2}$ is convex, hence (5) is fulfilled. Also, we readily check that the first three properties listed in Lemma 2 are satisfied by the elements of $\partial \operatorname{Prox}_{\varphi_{1}}(u), u \in U$. Thus, (7) holds.

$H$ has a unique root From Lemma 1 we infer by convexity that the unique solution $\bar{u}$ of the control reduced version of (OCP) corresponds to a unique root $\bar{q}$ of $H$.

Remark 7 If $\Omega_{\mathrm{obs}}=\Omega$ and $y_{d} \in Y$, then Lemma 6 is also true for the choices $Q:=H^{1}(I)^{N}$ and $P:=H^{1}\left(I ; H_{0}^{1}(\Omega)\right)$ as well as $Q:=C^{1}([0, T])^{N}$ and $P:=$ $C^{1}\left([0, T] ; L^{2}(\Omega)\right)$, cf. Remark 6. 
We obtain the following convergence result for Algorithm 1, in which we write $\nabla^{2} \hat{f}$ for the constant Hessian. Note in (2) and (3) that $\left(u^{k}\right)$ converges in various norms.

\section{Theorem 2}

1) Let $(\bar{y}, \bar{u}) \in Y \times U_{\mathrm{ad}}$ be the solution of (OCP), let $H$ be given by (8), and denote by $\bar{q} \in Q$ the unique root of $H$. Moreover, let $\mu \in(0,1)$. Then there exists $\varepsilon>0$ such that for every initial pair $\left(u^{0}, B_{0}\right) \in U \times \mathcal{L}(U, Q)$ with $\left\|u^{0}-\bar{u}\right\|_{U}<\epsilon$ and $\left\|B_{0}-\nabla^{2} \hat{f}\right\|_{\mathcal{L}(U, Q)}<\varepsilon$, Algorithm 1 either terminates after finitely many iterations or generates a sequence of iterates $\left(q^{k}\right)$ that converges $q$-linearly with rate $\mu$ to $\bar{q}$ in $Q$. If, in addition, $\sigma_{\min }, \sigma_{\max } \in(0,2)$ in Algorithm 1 and $\left(B_{0}-\nabla^{2} \hat{f}\right) \in \mathcal{L}(U, Q)$ is compact, then the convergence is $q$-superlinear.

2) If $\left(q^{k}\right)$ is generated by Algorithm 1 , then $\left(u^{k}\right)_{k \geq 1} \subset U_{\mathrm{ad}}$, i.e., every $u^{k}$ except possibly the starting point $u^{0}$ is feasible for (OCP). Moreover, $\left(u^{k}\right)_{k \geq 1},\{\bar{u}\} \subset$ $L^{\infty}(I)^{N}$ and there are $L_{y}, L_{p}>0$ such that

$$
\begin{array}{ll}
\left\|u^{k}-\bar{u}\right\|_{L^{s}(I)^{N}} \leq\left\|q^{k}-\bar{q}\right\|_{L^{s}(I)^{N}}, & \left\|u^{k}-\bar{u}\right\|_{L^{2}(I)^{N}} \leq T^{\frac{1}{2}}\left\|q^{k}-\bar{q}\right\|_{C([0, T])^{N}}, \\
\left\|y\left(u^{k}\right)-\bar{y}\right\|_{Y} \leq L_{y}\left\|q^{k}-\bar{q}\right\|_{Q}, & \left\|p\left(u^{k}\right)-\bar{p}\right\|_{P} \leq L_{p}\left\|q^{k}-\bar{q}\right\|_{Q}
\end{array}
$$

hold for all $k \geq 1$ and all $s \in[1, \infty]$.

If, in addition, $a, b \in Q$ holds, then we have $\left(u^{k}\right)_{k \geq 1},\{\bar{u}\} \subset Q$ and for all $k \geq 1$

$$
\left\|u^{k}-\bar{u}\right\|_{Q} \leq\left\|q^{k}-\bar{q}\right\|_{Q} .
$$

In particular, $\left\|q^{k}-\bar{q}\right\|_{Q} \rightarrow 0$ for $k \rightarrow \infty$ implies $\left\|u^{k}-\bar{u}\right\|_{Q} \rightarrow 0$.

3) If $\left(q^{k}\right)$ is generated by Algorithm 1 and converges $q$-linearly ( $q$-superlinearly) to $\bar{q}$ in $Q$, then $\left(u^{k}\right),\left(y\left(u^{k}\right)\right)$ and $\left(p\left(u^{k}\right)\right)$ converge r-linearly (r-superlinearly) in $L^{S}(I)^{N}$, respectively, $Y$ and $P$, where s is as in 2$)$. Moreover, $\left(H\left(q^{k}\right)\right)$ converges $r$-linearly ( $q$-superlinearly) in $Q$ to zero, then.

Proof Proof of 1) The claim of 1) follows from Theorem 1, part 1), which can be applied since Assumption 1 is satisfied, cf. Lemma 6.

Proof of 2) The feasibility of the $u^{k}$ is valid since $\operatorname{Prox}_{\varphi_{1}}(Q) \subset U_{\text {ad }}$ and since $u^{k}=\operatorname{Prox}_{\varphi_{1}}\left(q^{k}\right)$ for $k \geq 1$. Moreover, the property $\left(u^{k}\right)_{k \geq 1},\{\bar{u}\} \subset L^{\infty}(I)^{N}$ follows from $U_{\text {ad }} \subset L^{\infty}(I)^{N}$. To show the first inequality, we remark that $q^{k}, \bar{q} \in Q$ implies $q^{k}, \bar{q} \in L^{s}(I)^{N}$ for all $s \in[1, \infty]$ and all $k \geq 0$. It is straightforward to infer for $q, \bar{q} \in L^{s}(I)^{N}$ that $\left|\rho_{i}\left(q_{i}(t)\right)-\rho_{i}\left(\bar{q}_{i}(t)\right)\right| \leq\left|q_{i}(t)-\bar{q}_{i}(t)\right|$ for a.e. $t \in I, 1 \leq$ $i \leq N$. The same can be established for $\rho_{i}$ replaced by $\left(\Pi_{U_{\mathrm{ad}}}\right)_{i}$. Together, this implies $\left|u_{i}(t)-\bar{u}_{i}(t)\right| \leq\left|q_{i}(t)-\bar{q}_{i}(t)\right|$ for a.e. $t \in I, 1 \leq i \leq N$, proving the first error bound in 2). Moreover, this also implies (10) provided that $\left(\Pi_{U_{\text {ad }}} \circ \sigma\right)(Q) \subset Q$ if $a, b \in Q$. This property of $\Pi_{U_{\text {ad }}} \circ \sigma$ is elementary to see, for instance by showing it separately for $\sigma$ and $\Pi_{U_{\text {ad }}}$. The second error bound follows from the first for $s=2$ by use of $\left\|q^{k}-\bar{q}\right\|_{L^{2}(I)^{N}} \leq|I|^{\frac{1}{2}}\left\|q^{k}-\bar{q}\right\|_{C([0, T])^{N}}$, where $|I|$ is the Lebesgue measure of $I$. Since $u \mapsto y(u)$ and $u \mapsto p(u)$ are linear and continuous from $U$ to $Y=P$, they are globally Lipschitz, too. This in combination with the estimate $\left\|u^{k}-\bar{u}\right\|_{L^{2}(I)^{N}} \leq\left\|q^{k}-\bar{q}\right\|_{L^{2}(I)^{N}}$ and the continuous embedding $Q \hookrightarrow L^{2}(I)^{N}$ yields the third and fourth error bound. 
Proof of 3) The claims follow from the error estimates in 2) and, for $\left(H\left(q^{k}\right)\right)$, from part 3) of Corollary 1.

Remark 8 It is not difficult to argue that Theorem 2 also holds for $Q:=L^{\hat{s}}(I)^{N}$ for any $\hat{s} \in(2, \infty]$. Of course, the $L^{s}$ estimates of that theorem are then only true for $s \in[1, \hat{s}]$. The use of a weaker norm in $Q$ relaxes the assumption on $\left(u^{0}, B_{0}\right)$ and the compactness requirement in that theorem.

In Theorem 2 we have worked with $Q=C([0, T])^{N}, P=W\left(I ; L^{2}(\Omega), H_{0}^{1}(\Omega)\right)$, and $y_{\mathrm{d}} \in L^{2}\left(I \times \Omega_{\mathrm{obs}}\right)$, and we have allowed $\Omega_{\mathrm{obs}} \neq \Omega$. If $\Omega_{\mathrm{obs}}=\Omega$ and $y_{\mathrm{d}}$ is more regular, then we can exploit this in two ways. First, by letting $Q$ and $P$ be spaces of smoother functions the convergence results of Theorem 2, that contain the norms of $Q$ and $P$, become stronger (but a better quality of the initial approximation $\left(u^{0}, B_{0}\right)$ is required for the theorem to apply). Second, the operator $\nabla^{2} \hat{f}(\bar{u})$ becomes compact. In view of Theorem 2, part 1), this suggests to choose a compact initial $B_{0}$ to achieve q-superlinear convergence, for instance $B_{0}=0$. In contrast, $B_{0}=\Theta I$ for $\Theta>0$ can only be expected to yield q-linear convergence, presumably the faster the smaller $\Theta$ is. Indeed, the numerical results in Sect. 6 show that $B_{0}=0$ is most often superior to scaled identities and that the results for $B_{0}=\Theta I$ improve as $\Theta$ becomes smaller. The following two lemmas offer several choices for $P$ and $Q$ and provide the corresponding convergence results. For simplicity we consider constant bounds.

Lemma 7 Let $Q:=H^{1}(I)^{N}, U:=L^{2}(I)^{N}, Y:=W\left(I ; L^{2}(\Omega), H_{0}^{1}(\Omega)\right)$, and $P:=$ $H^{1}\left(I ; H_{0}^{1}(\Omega)\right)$. Suppose that $\Omega_{\mathrm{obs}}=\Omega$ and $y_{d} \in Y$, and let $a_{i}, b_{i}$ be constant for each $1 \leq i \leq N$. Then all claims of Theorem 2 except (10) are true. If $Q:=L^{\infty}(I)^{N}$ or $Q:=C([0, T])^{N}$ is used instead, then all claims of Theorem 2 are true and the operator $\nabla^{2} \hat{f} \in \mathcal{L}(U, Q)$ is compact.

Proof Prooffor $Q:=H^{1}(I)^{N}$ The proof is completely analogue to the one of Theorem 2, except for the claim above (10) and the one below (10). That is, we have to establish that $\left(u^{k}\right)_{k \geq 1},\{\bar{u}\} \subset Q=H^{1}(I)^{N}$ and $u^{k} \rightarrow \bar{u}$ in $Q$ provided that $q^{k} \rightarrow \bar{q}$ in $Q$. In fact, this follows since $\operatorname{Prox}_{\varphi_{1}}=\Pi_{U_{\text {ad }}} \circ \sigma$ satisfies $\operatorname{Prox}_{\varphi_{1}}(Q) \subset Q$ and since $\operatorname{Prox}_{\varphi_{1}}: Q \rightarrow Q$ is continuous. Both properties can be proven separately for $\Pi_{U_{\mathrm{ad}}}$ and $\sigma$. For $\Pi_{U_{\mathrm{ad}}}$ these properties follow from (Kinderlehrer and Stampacchia 2000, Chapter II, Corollary A.5), which shows that $\Pi_{U_{\text {ad }}}\left(W^{1, s}(I)^{N}\right) \subset W^{1, s}(I)^{N}$ for all $s \in[1, \infty]$, and from (Appell and Zabrejko 1990, Theorem 9.5). Using the fact that cut-off does not increase the $H^{1}$-norm, cf. (Kinderlehrer and Stampacchia 2000, Theorem A.1), the corresponding proof for $\sigma$ is elementary.

Proof for $Q:=L^{\infty}(I)^{N}$ and for $Q:=C([0, T])^{N}$ The compactness of $\nabla^{2} \hat{f}$ follows from the compactness of $H^{1}(I)^{N} \hookrightarrow L^{\infty}(I)^{N}$, respectively, of $H^{1}(I)^{N} \hookrightarrow$ $C([0, T])^{N}$. All other claims can be established as in the proof of Theorem 2 .

Still higher regularity is available in the situation of Lemma 7.

Lemma 8 Let $Q:=C^{0, \hat{s}}([0, T])^{N}, \hat{s} \in(0,1], U:=L^{2}(I)^{N}, Y:=W\left(I ; L^{2}(\Omega)\right.$, $\left.H_{0}^{1}(\Omega)\right)$, and $P:=C^{1}\left([0, T] ; L^{2}(\Omega)\right)$. Suppose that $\Omega_{\mathrm{obs}}=\Omega$ and $y_{d} \in Y$, and let $a_{i}, b_{i}$ be constant for each $1 \leq i \leq N$. Then all claims of Theorem 2 except (10) and the convergence assertion below it are true. Moreover, $q^{k} \rightarrow \bar{q}$ in $Q$ implies $u^{k} \rightarrow \bar{u}$ 
in $C^{0, \hat{s}-\tau}([0, T])^{N}$ for any $\tau \in(0, \hat{s})$ and for $\hat{s} \neq 1$ the operator $\nabla^{2} \hat{f} \in \mathcal{L}(U, Q)$ is compact.

Proof The main task is to establish that $\operatorname{Prox}_{\varphi_{1}}=\Pi_{U_{\text {ad }}} \circ \sigma$ satisfies $\operatorname{Prox}_{\varphi_{1}}(Q) \subset Q$ and that $\operatorname{Prox}_{\varphi_{1}}: Q \rightarrow C^{0, \hat{s}-\tau}([0, T])^{N}$ is continuous at $\bar{q}$ for any $\tau \in(0, \hat{s})$. The proof can be undertaken separately for $\Pi_{U_{\text {ad }}}$ and $\sigma$. The property $\operatorname{Prox}_{\varphi_{1}}(Q) \subset Q$ follows from (Appell and Zabrejko 1990, Theorem 7.1), and the continuity at $\bar{q}$ is a consequence of (Appell and Zabrejko 1990, first part of Theorem 7.6). The compactness of $\nabla^{2} \hat{f} \in \mathcal{L}(U, Q)$ is implied by the fact that $C^{0,1}([0, T])^{N} \hookrightarrow C^{0, \hat{s}}([0, T])^{N}$ is compact for $\hat{s} \in(0,1)$.

\subsubsection{The semilinear heat equation}

We show that our method also applies to nonlinear state equations. To this end, we consider the same setting as in Sect. 4.2.1 but replace the linear heat equation in (OCP) by a semilinear variant. For ease of presentation we use the rather concrete

$$
\begin{cases}y_{t}-\Delta y+m_{0}(t, x)+m_{1}(t, x) y^{2 \kappa+1}=\sum_{i=1}^{N} g_{i}(x) u_{i}(t) & \text { in } I \times \Omega \\ y=0 & \text { on } \Sigma \\ y(0, x)=y_{0}(x) & \text { in } \Omega\end{cases}
$$

defined on the nonempty and bounded Lipschitz domain $\Omega \subset \mathbb{R}^{d}, 1 \leq d \leq 3$, and the time interval $I=(0, T), T>0$. Here, $\kappa \in \mathbb{N}, y_{0} \in L^{\infty}(\Omega), g_{i} \in L^{\overline{2}}(\Omega)$ for all $1 \leq i \leq N$ with support $\omega_{i} \subset \Omega, m_{0} \in L^{2}(I \times \Omega), y_{d} \in L^{p}(I \times \Omega)$ with $p>1+\frac{d}{2}$ for $d>1$ and $p \geq 2$ for $d=1$, and $m_{1} \in L^{\infty}(I \times \Omega)$ is nonnegative. We set $Y:=W\left(I ; L^{2}(\Omega), H_{0}^{1}(\Omega)\right) \cap L^{\infty}(I \times \Omega)$ and $P:=Y \cap C([0, T] \times \bar{\Omega})$. For convenience let us recall that $N \in \mathbb{N}, Q=C([0, T])^{N}, U=L^{2}(I)^{N}$. The set of admissible controls is still given by (7) with $a, b \in L^{\infty}(I)^{N}, a \leq b$ a.e. in $I$. From (Casas et al. 2017, Proposition 2.1) we obtain that for every $u \in U$ there exists a unique $y=y(u) \in Y$ such that (sem) is satisfied. The solution operator $u \mapsto y(u)$ is $C^{2}$ from $U$ to $Y$ by the implicit function theorem, cf. also (Casas et al. 2017, Proposition 2.2). Thus, $\hat{f}(u):=\frac{1}{2}\left\|y(u)-y_{\mathrm{d}}\right\|_{L^{2}\left(I \times \Omega_{\mathrm{obs}}\right)}^{2}$ is continuously differentiable from $U$ to $\mathbb{R}$. Since the control reduced problem $(\mathrm{P})$ does no longer have a convex objective function, it is possible that $(\mathrm{P})$ has multiple local and global minimizers; it is, however, standard to show that at least one global minimizer $\bar{u} \in U_{\text {ad }}$ exists, for instance by following the arguments of (Tröltzsch 2010, Theorem 5.7). The associated state is $\bar{y}:=y(\bar{u}) \in Y$. Similarly to Lemma 5 the following holds.

Lemma 9 For the control reduced version of (OCP) with (sem) the mapping $H$ defined in (3) is for $\gamma=1$ given by

$H: Q \rightarrow Q, \quad H_{i}(q)(t)=\int_{\omega_{i}} g_{i}(x) p\left(\Pi_{U_{\mathrm{ad}}}(\sigma(q))\right)(t, x) d x+\alpha_{i} q_{i}(t), \quad 1 \leq i \leq N$ 
Here, the adjoint state $p=p(u) \in P$ uniquely solves the linear problem

$$
\begin{cases}-p_{t}-\Delta p+(2 \kappa+1) m_{1}(t, x) y^{2 \kappa} p=\chi_{I \times \Omega_{\mathrm{obs}}} \cdot\left(y(u)-y_{d}\right) & \text { in } I \times \Omega \\ p=0 & \text { on } \Sigma, \\ p(T)=0 & \text { in } \Omega\end{cases}
$$

Proof Adjoint calculus yields $(\nabla \hat{f}(u))_{i}(t)=\int_{\omega_{i}} g_{i}(x) p(t, x) / \alpha_{i} \mathrm{~d} x$ for $1 \leq i \leq$ $N$. Inserting this in $H(q)=\nabla \hat{f}\left(\operatorname{Prox}_{\varphi_{1}}(q)\right)+q$ and using Lemma 3 establishes the formula for $H$. Moreover, the assumptions on the problem data together with $y \in Y \subset L^{\infty}(I \times \Omega)$ imply $p(u) \in P$, cf. (Tröltzsch 2010, Theorem 5.5). From $p \in P \subset C([0, T] \times \bar{\Omega})$ it follows that $H$ maps to $Q$.

Assumption 1 holds for the semilinear problem under the following condition.

Lemma 10 Assumption 1 holds for the control reduced version of (OCP) with state equation (sem) provided

$$
2 \kappa(2 \kappa+1) m_{1} y(\bar{u})^{2 \kappa-1} p(\bar{u}) \leq 1 \quad \text { pointwise a.e. in } I \times \Omega_{\mathrm{obs}} \text {. }
$$

Proof Conditions 1)-4) of Assumption 1 were already established, cf. the remarks above Lemma 9 and those above Lemma 5. In the proof of Lemma 9 we have demonstrated that $\nabla \hat{f}$ maps $U$ to $Q$. As $u \mapsto p(u)$ is $C^{2}$ from $U$ to $P$, it is not hard to deduce that $\nabla \hat{f}: U \rightarrow Q$ is $C^{2}$, so (5) is satisfied. Condition 6) follows from Lemma 4. To establish (7) we use Lemma 2. Except for (5) the assumptions of Lemma 2 follow as in the proof of Lemma 6. A computation reveals that $\left(\nabla^{2} \hat{f}(\bar{u}) h, h\right)_{U}=$ $\left(y^{\prime}(\bar{u}) h, r y^{\prime}(\bar{u}) h\right)_{U}$ for all $h \in U$, where $r:=1-2 \kappa(2 \kappa+1) m_{1} y(\bar{u})^{2 \kappa-1} p(\bar{u})$. This shows that (5) is fulfilled for $v:=\gamma$. Thus, 7) holds.

We obtain the following convergence result for Algorithm 1. Note in (2) and (3) that $\left(u^{k}\right)$ converges in various norms.

Theorem 3 1) Let $(\bar{y}, \bar{u}) \in Y \times U_{\text {ad }}$ be a solution of (OCP) with (sem) as state equation and suppose that (11) is satisfied. Let $H$ be defined according to Lemma 9 and denote $\bar{q}:=-\nabla \hat{f}(\bar{u}) \in Q$. Moreover, let $\mu \in(0,1)$. Then there exists $\varepsilon>0$ such that for every initial pair $\left(u^{0}, B_{0}\right) \in U \times \mathcal{L}(U, Q)$ with $\left\|u^{0}-\bar{u}\right\|_{U}<\epsilon$ and $\left\|B_{0}-\nabla^{2} \hat{f}\left(u^{0}\right)\right\|_{\mathcal{L}(U, Q)}<\varepsilon$, Algorithm 1 either terminates after finitely many iterations or generates a sequence of iterates $\left(q^{k}\right)$ that converges $q$-linearly with rate $\mu$ to $\bar{q}$ in $Q$. If, in addition, $\sigma_{\min }, \sigma_{\max } \in(0,2)$ in Algorithm 1 and $\left(B_{0}-\nabla^{2} \hat{f}(\bar{u})\right) \in \mathcal{L}(U, Q)$ is compact, then the convergence is q-superlinear.

2) If $\left(q^{k}\right)$ is generated by Algorithm 1 , then $\left(u^{k}\right)_{k \geq 1} \subset U_{\mathrm{ad}}$, i.e., every $u^{k}$ except possibly the starting point $u^{0}$ is feasible for (OCP). Moreover, $\left(u^{k}\right)_{k \geq 1},\{\bar{u}\} \subset$ $L^{\infty}(I)^{N}$, and if $\lim _{k \rightarrow \infty}\left\|q^{k}-\bar{q}\right\|_{Q}=0$ then there are $L_{y}, L_{p}>0$ such that

$$
\begin{array}{ll}
\left\|u^{k}-\bar{u}\right\|_{L^{s}(I)^{N}} \leq\left\|q^{k}-\bar{q}\right\|_{L^{s}(I)^{N}}, & \left\|u^{k}-\bar{u}\right\|_{U} \leq T^{\frac{1}{2}}\left\|q^{k}-\bar{q}\right\|_{Q}, \\
\left\|y\left(u^{k}\right)-\bar{y}\right\|_{Y} \leq L_{y}\left\|q^{k}-\bar{q}\right\|_{Q}, & \left\|p\left(u^{k}\right)-\bar{p}\right\|_{P} \leq L_{p}\left\|q^{k}-\bar{q}\right\|_{Q}
\end{array}
$$


hold for all $k \geq 1$ and all $s \in[1, \infty]$.

If, in addition, $a, b \in Q$ holds, then we have $\left(u^{k}\right)_{k \geq 1},\{\bar{u}\} \subset Q$ and for all $k \geq 1$

$$
\left\|u^{k}-\bar{u}\right\|_{Q} \leq\left\|q^{k}-\bar{q}\right\|_{Q}
$$

3) If $\left(q^{k}\right)$ is generated by Algorithm 1 and converges $q$-linearly ( $q$-superlinearly) to $\bar{q}$ in $Q$, then $\left(u^{k}\right),\left(y\left(u^{k}\right)\right)$ and $\left(p\left(u^{k}\right)\right)$ converge $r$-linearly ( $r$-superlinearly) in $L^{\infty}(I)^{N}$, respectively, $Y$ and $P$. Moreover, $\left(H\left(q^{k}\right)\right)$ converges r-linearly $(q$ superlinearly) in $Q$ to zero, then.

Proof Proof of 1) The claim of 1) follows from Theorem 1, part 1), which can be applied since Assumption 1 is satisfied, cf. Lemma 10.

Proof of 2) The proof is almost identical to the one for part 2) of Theorem 2. The only necessary change is that $u \mapsto y(u)$ and $u \mapsto p(u)$ are no longer globally Lipschitz, but only locally Lipschitz. The existence of $L_{y}$ and $L_{p}$ thus follows from $u^{k} \rightarrow \bar{u}$ in $U$, the latter being a consequence of the assumption that $q^{k} \rightarrow \bar{q}$ in $Q$.

Proof of 3) The claims follow from the error estimates in 2) and, for $\left(H\left(q^{k}\right)\right)$, from part 3) of Corollary 1.

\section{Implementation}

The hybrid framework is tested with three different quasi-Newton updates: Broyden, SR1, and BFGS. The methods are implemented with limited-memory techniques storing the last up to $L$ (called the limit) updates as vectors and matrix-free. Consequently, the Newton systems are solved with iterative methods, specifically with GMRES or cg. The limited-memory BFGS method is implemented in the compact variant according to Byrd et al. (1994), see also (Nocedal and Wright 2006, (7.24)). Additionally, the quasi-Newton methods are compared to Newton's method itself, i.e., setting $B_{k}=\nabla^{2} \hat{f}\left(u^{k}\right)$ for all $k$ and dropping lines 8-11 in Algorithm 1. Here, the matrix-free evaluation in a direction is implemented via forward-backward solve. We stress that when Newton's method is used, Algorithm 1 is a standard semismooth Newton method.

The methods are applied with three different globalization techniques. First, a standard backtracking line search on the residual norm $\|H\|_{U}$ is used together with GMRES (ls-GMRES). The line search selects the smallest integer $0 \leq j \leq 18$ with $\left\|H\left(q^{k}+0.5^{j} s^{k}\right)\right\|_{U}<\left\|H\left(q^{k}\right)\right\|_{U}$, and $j=19$ otherwise. GMRES from MATLAB is used with a tolerance of $10^{-10}$ and a maximum number of 50 iterations to solve the full Newton system. Second, a non-monotone line search (nls-GMRES) with $N_{\mathrm{ls}} \in \mathbb{N}$ steps is used, where the step size $\rho^{j}=0.5^{j}, 0 \leq j \leq 18$, is accepted as soon as $R\left(q^{k}+\rho^{j} s^{k}\right)<\max \left\{R\left(q^{k}\right), R\left(q^{k-1}\right), \ldots, R\left(q^{\bar{k}-N_{\mathrm{ls}}+1}\right)\right\}$ holds, and $j=19$ otherwise. Therein, $R$ will be the residual norm $\|H(\cdot)\|_{U}$ or the objective $f(\cdot)+\varphi(\cdot)$ of (P). Third, a trust-region method is investigated based on Steihaug-cg (tr-cg), cf. Steihaug (1983). The precise algorithm is included in "Appendix A". It is started with a radius $\varrho_{0}=0.1$ and stopped with a relative tolerance of $10^{-5}$. The parameters are $\sigma_{1}=0.05, \sigma_{2}=0.25, \sigma_{3}=0.7$, radius factors $f_{1}=0.4, f_{2}=2, f_{3}=0.6$, and a 
maximum radius $\varrho_{\max }=2$. Up to 300 iterations are allowed. The update of the quasiNewton matrix is also carried out at rejected steps. Additionally, in case of BFGS, the update is only applied if the curvature condition $\left(y^{k}, s_{u}^{k}\right)_{U}>0$ holds. To be able to use Steihaug-cg, the linear system is first reduced to a symmetric one by restricting to the Hilbert space induced by the inner product $\left(\cdot, M_{k} \cdot\right)_{U}$ with $M_{k} \in \partial \operatorname{Prox}_{\varphi_{\gamma}}\left(q^{k}\right)$, cf. Lemma 2 and (Pieper 2015, Def. 3.4). Then a correction step gives the full update, cf. (Pieper 2015, (3.25)). The cg method is limited to 100 iterations and stopped with a relative tolerance of $10^{-10}$. We use small tolerances to suppress the influence of inexact linear system solves.

\section{Numerical experiments}

The numerical experiments below show the application of the hybrid method to optimal control problems. The first example is the heat equation, the second the bilinear control of the Bloch equation in magnetic resonance imaging. As nonsmooth problem parts we deal with pointwise box constraints on the controls and sparsity promoting objectives. All computations are carried out using MATLAB 2017a and a workstation with two Intel Xeon X5675 (24GB RAM, twelve cores with 3.06GHz). All time measurements are performed on a single CPU without multi-threading.

\subsection{Time-dependent tracking of the heat equation}

The first example problem is sparse control of the linear heat equation with $\Omega_{\mathrm{obs}}=$ $\Omega=(-1,1)^{2}$ and time domain $I=(0,1)$. Specifically, we consider

$$
\begin{aligned}
\min _{(y, u) \in Y \times U_{\mathrm{ad}}} \frac{1}{2}\left\|y-y_{\mathrm{d}}\right\|_{L^{2}\left(I \times \Omega_{\mathrm{obs}}\right)}^{2}+\frac{\alpha}{2}\|u\|_{L^{2}(I)}^{2}+\beta\|u\|_{L^{1}(I)} \\
\text { s.t. } \begin{cases}y_{t}-\Delta y=\chi_{\omega}(x) u(t) & \text { in } I \times \Omega, \\
\partial_{\nu} y=0 & \text { on } \Sigma, \\
y(0, x)=y_{0}(x) & \text { in } \Omega .\end{cases}
\end{aligned}
$$

Therein, $U_{\mathrm{ad}}=\{u \in U: a \leq u(t) \leq b$ for a.e. $t \in I\}$ with $a=-1$ and $b=1$, $y_{\mathrm{d}}(t, x)=2 \sin (2 \pi t), \alpha>0, \beta \geq 0, \chi_{\omega}(x) \in L^{\infty}(\Omega)$ is the characteristic function of the right half $\omega=(0,1) \times(-1,1)$, and $y_{0} \equiv 0$. The example is a special case of (OCP) with $N=1$, however using Neumann instead of Dirichlet boundary conditions. We emphasize that the results of Sect. 4.2 can also be developed for these boundary conditions. In view of Lemmas 7 and 8 we expect convergence in rather strong norms. Specifically, we are interested in q-superlinear convergence of $\left(q^{k}\right)$ in $H^{1}(I)$ and in $C^{0, s}([0, T])$ for all $s \in(0,1)$, convergence of $\left(u^{k}\right)$ in $H^{1}(I)$ and in $C^{0, s-\tau}([0, T])$ for all $\tau \in(0, s)$, and r-superlinear convergence of $\left(u^{k}\right)$ in $L^{2}(I)$ and $L^{\infty}(I)$. Furthermore, we should be able to observe the error estimates $\left\|u^{k}-\bar{u}\right\|_{L^{s}(I)} \leq\left\|q^{k}-\bar{q}\right\|_{L^{s}(I)}$ for $s \in\{2, \infty\}$, cf. part 2) of Theorem 2. We will investigate these properties numerically.

We use an unstructured triangular mesh with 725 P1 elements generated by MATLAB's initmesh with Hmax=0.1, see Fig. 1. As time-stepping scheme the CG(1) 

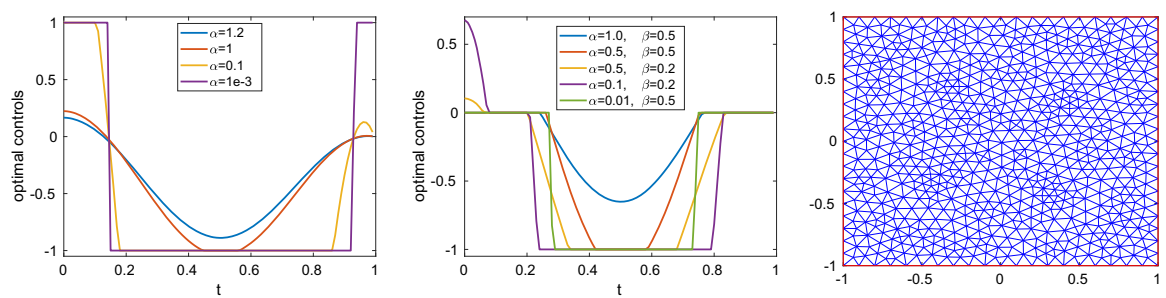

Fig. 1 Optimal control $\bar{u}$ for different $\alpha$ with $\beta=0$ (left), with $\beta>0$ (mid), and domain $\Omega$ with triangulation (right)

Table 1 Iterations of the quasi-Newton implementations for different $\alpha$ (rows) and for different $B_{0}$ (columns per method) with $\beta=0 .|\mathcal{A}|$ is the number of control components on the bounds out of $101,-$ means no convergence within 50 iterations

\begin{tabular}{|c|c|c|c|c|c|c|c|c|c|c|c|c|c|c|}
\hline \multirow[t]{2}{*}{$\alpha$} & \multirow{2}{*}{$|\mathcal{A}|$} & \multirow{2}{*}{ SN } & \multicolumn{4}{|c|}{ Broyden } & \multicolumn{4}{|c|}{ SR1 } & \multicolumn{4}{|c|}{ BFGS } \\
\hline & & & $\overline{\alpha I}$ & $\frac{y^{T} s}{s^{T} s} I$ & $\frac{y^{T} y}{y^{T} S} I$ & 0 & $\overline{\alpha I}$ & $\frac{y^{T} s}{s^{T} s} I$ & $\frac{y^{T} y}{y^{T} s} I$ & 0 & $\overline{\alpha I}$ & $\frac{y^{T} s}{s^{T} s} I$ & $\frac{y^{T} y}{y^{T} s} I$ & $I$ \\
\hline $10^{0}$ & 12 & 2 & 12 & 10 & 10 & 6 & 8 & 9 & 10 & 5 & 12 & 7 & 9 & 12 \\
\hline $10^{-1}$ & 80 & 4 & 12 & 15 & - & 7 & 10 & 17 & - & 6 & 14 & 11 & 14 & 18 \\
\hline $10^{-2}$ & 95 & 6 & 11 & 19 & - & 7 & 9 & - & - & 7 & 10 & 13 & 20 & 22 \\
\hline $10^{-3}$ & 99 & 11 & 10 & 17 & - & 9 & 9 & - & - & 12 & 9 & 12 & 12 & 14 \\
\hline
\end{tabular}

method is chosen (corresponding to the Crank-Nicolson method) with 101 equidistant time points and a piecewise constant discretization of $q$ and $u$. We initialize the algorithm with $u^{0}=0$, corresponding to $q^{0}=0$. Since the problem is strongly convex, the solution is possible based on the simple globalization ls-GMRES (for $\beta=0$ and sufficiently large $\alpha$ we expect based on a global convergence result in Kunisch and Rösch (2002, Theorem 3) that it would even be possible to drop the line search and use only full steps). The optimal controls for different $\alpha, \beta$ are depicted in Fig. 1 . We will use varying parameters $\alpha, \beta$, with $\alpha=0.01$ as default value. For the limit we use $L=30$.

The proximal mapping is $\Pi_{U_{\mathrm{ad}}}(\sigma(q))$ from Lemma 3. In the algorithm we select the differential $M_{k} \in \partial\left(\Pi_{U_{\text {ad }}} \circ \sigma\right)\left(q^{k}\right)$ that satisfies, for all $h \in Q,\left(M_{k} h\right)(t)=$ $h(t)$ if $\left|q^{k}(t)\right| \geq \beta / \alpha$ and $a<\sigma\left(q^{k}\right)(t)<b$, and $\left(M_{k} h\right)(t)=0$ otherwise. In (6) this corresponds to the choice $r(t)=1$ for all $t$ satisfying $\left|q^{k}(t)\right|=\beta / \alpha$ and $a<\sigma\left(q^{k}\right)(t)<b$, and $r(t)=0$ for all remaining $t \in I$.

The first study shows the performance of the optimization methods for different initializations of $B_{0}$. Table 1 shows the results for $\beta=0$. The first two columns show $\alpha$ and the resulting number of active points $|\mathcal{A}|$ (optimal control on upper or lower bound) out of 101. The other columns depict the iteration numbers that are needed to reach the relative tolerance $10^{-8}$, separately for semismooth Newton (SN) and the three hybrid methods. Here, four different initializations are applied, including the scaled identity $\alpha I$, the zero matrix 0 (except for BFGS, where this produces a vanishing denominator), and the two formulas (Byrd et al. 1994, Eq.(3.23)) $B_{0}=$ $y^{T} s /\left(s^{T} s\right) I$ and (Nocedal and Wright 2006, Eq.(7.20)) $B_{0}=y^{T} y /\left(y^{T} s\right) I$. We note 
Table 2 Iteration counts for different $\alpha$ and $\beta .|\mathcal{A}|$ and $|\mathcal{O}|$ denote the number out of 101 control points that are on the bounds, respectively, that are zero

\begin{tabular}{lcrllllc}
\hline$\alpha$ & $\beta$ & $|\mathcal{A}|$ & $|\mathcal{O}|$ & $\mathrm{SN}$ & $\mathrm{Br}$ & $\mathrm{SR} 1$ & $\mathrm{BFGS}$ \\
\hline 1.00 & 0.1 & 0 & 18 & 4 & 6 & 5 & 8 \\
0.10 & 0.1 & 68 & 20 & 4 & 8 & 6 & 12 \\
0.01 & 0.1 & 79 & 20 & 6 & 6 & 6 & 13 \\
1.00 & 0.2 & 0 & 34 & 3 & 6 & 5 & 7 \\
0.10 & 0.2 & 56 & 30 & 4 & 8 & 7 & 12 \\
0.01 & 0.2 & 68 & 31 & 5 & 7 & 6 & 17 \\
1.00 & 0.5 & 0 & 48 & 3 & 5 & 5 & 7 \\
0.10 & 0.5 & 41 & 53 & 3 & 6 & 5 & 8 \\
0.01 & 0.5 & 45 & 53 & 5 & 7 & 6 & 9 \\
1.00 & 1.0 & 0 & 71 & 3 & 4 & 4 & 6 \\
0.10 & 1.0 & 14 & 77 & 4 & 6 & 5 & 10 \\
0.01 & 1.0 & 21 & 77 & 5 & 7 & 6 & 9 \\
\hline
\end{tabular}

that both formulas are implemented in the first step with $B_{0}=0$ for Broyden/SR1 and $B_{0}=\alpha I$ for BFGS. A good performance for any $\alpha$ and for all three methods can be obtained by choosing $B_{0}=\alpha I$. However, Broyden and SR1 show faster convergence with the zero initialization, and BFGS shows in the mean less iterations with $B_{0}=y^{T} s /\left(s^{T} s\right) I$, which are the default initializations for all other studies below. We stress that from an infinite-dimensional point of view the choice $B_{0}=0$ results in $B_{0}-\nabla^{2} \hat{f}(\bar{u}) \in \mathcal{L}(U, Q)$ being compact, cf. Lemma 7 and Lemma 8, whereas this is not the case for the scaled identities. Therefore, BFGS may be inherently slower than Broyden and SR1 in the following experiments, cf. also the reasoning provided above Lemma 7. This will indeed turn out to be true.

The results for $\beta>0$ are depicted in Table 2. Here, $|\mathcal{O}|$ collects the number of time points with zero control out of 101 . We note that the inequality constraints are in general inactive $(|\mathcal{A}|=0)$ in the optimum if $\alpha \geq 1$. On the other hand, smaller values $\alpha \leq 0.01$ result in only one or two inactive points (the number of inactive points is $101-|\mathcal{A}|-|\mathcal{O}|)$. Increasing the parameter $\beta$ between 0.1 and 1 increases the sparsity from around $20 \%$ to $80 \%$. For $\beta \geq 2$ the optimal solution is zero. The four last columns show the iteration counts of the four methods with default initializations. All methods convergence quickly for all values of $\alpha$ and $\beta$. They tend to require fewer iterations for larger $\beta$, which corresponds to more degrees of freedom being fixed to zero. The desired tolerance is reached after at most 8 iterations for Broyden $(\mathrm{Br})$, respectively, 7 iterations for SR1. BFGS needs up to 17 iterations.

The iteration counts of the hybrid methods for different discretizations are depicted in the upper part of Table 3 for $\alpha=0.01, \beta=0$ using $N_{c}+1$ equidistant time points and three different meshes from initmesh with $N_{x}$ nodes. The results show mesh independence for all three quasi-Newton methods with respect to both the spatial and the temporal discretization. The lower part of the table shows the corresponding runtimes in seconds. All values are averages of five runs. Broyden and SR1 show nearly identical runtimes in this example and are twice as fast as BFGS. All three methods outperform the semismooth Newton method in runtime. For all time and 
Table 3 Iterations and runtimes (in sec.) for different discretizations with $\alpha=0.01, \beta=0$

\begin{tabular}{|c|c|c|c|c|c|c|c|c|c|c|c|c|}
\hline \multirow{2}{*}{$\begin{array}{l}\text { Iterations } \\
N_{c} \backslash N_{x}\end{array}$} & \multicolumn{3}{|l|}{ SN } & \multicolumn{3}{|c|}{ Broyden } & \multicolumn{3}{|l|}{ SR1 } & \multicolumn{3}{|c|}{ BFGS } \\
\hline & 725 & 1938 & 7701 & 725 & 1938 & 7701 & 725 & 1938 & 7701 & 725 & 1938 & 7701 \\
\hline 100 & 6 & 6 & 6 & 7 & 7 & 7 & 7 & 7 & 7 & 13 & 13 & 13 \\
\hline 400 & 5 & 5 & 5 & 7 & 8 & 7 & 7 & 7 & 7 & 14 & 14 & 14 \\
\hline 1600 & 5 & 5 & 5 & 7 & 7 & 7 & 7 & 7 & 7 & 14 & 14 & 14 \\
\hline 6400 & 5 & 5 & 5 & 7 & 8 & 7 & 7 & 7 & 7 & 14 & 14 & 14 \\
\hline \multicolumn{13}{|l|}{ Runtimes } \\
\hline 100 & 11 & 40 & 210 & 2 & 5 & 33 & 2 & 6 & 31 & 3 & 9 & 56 \\
\hline 400 & 40 & 128 & 681 & 6 & 22 & 112 & 6 & 20 & 111 & 12 & 37 & 211 \\
\hline 1600 & 158 & 486 & 2755 & 27 & 83 & 446 & 27 & 82 & 449 & 51 & 154 & 841 \\
\hline 6400 & 643 & 1971 & 10855 & 137 & 456 & 2095 & 148 & 419 & 2102 & 290 & 763 & 3945 \\
\hline
\end{tabular}

The rows show results for $N_{c}$ control points. The three columns per method show results for triangulations with $N_{x}=725,1938,7701$ nodes. All runtimes are mean values of five runs

space discretizations a speedup factor of five to six is observed for Broyden and SR1, and three to four for BFGS.

The next study analyzes the superlinear convergence properties numerically. For comparison the optimal solution $\bar{q}$ is first computed in high precision with the semismooth Newton method and GMRES using fine relative tolerances of $10^{-14}$ for both. Then the indicators of superlinear convergence

$$
r_{u}^{k}:=\frac{\left\|u^{k+1}-\bar{u}\right\|_{Z}}{\left\|u^{k}-\bar{u}\right\|_{Z}} \quad \text { and } \quad r_{q}^{k}:=\frac{\left\|q^{k+1}-\bar{q}\right\|_{Z}}{\left\|q^{k}-\bar{q}\right\|_{Z}}
$$

are computed for each method for the norms $Z=L^{2}(I), L^{\infty}(I)$ and the seminorms $Z=H^{1}(I), C^{0,1}([0, T])$. For q-superlinear convergence these indicators should converge to zero in the last steps of an optimization run. Table 4 depicts the indicators for the last four iterations. The results are obtained for $\alpha=0.1, \beta=0.2$, and a relative tolerance of $10^{-8}$. We observe that the semismooth Newton method converges in one step as soon as the active set has converged. Broyden and SR1 show fast superlinear convergence with final indicators between $7 \times 10^{-3}$ and $6 \times 10^{-4}$ both for the control $u$ and the optimization variable $q$. For BFGS the indicators are slightly larger, but also decrease towards the end. If $\alpha$ is further reduced to 0.01 , we observe one-step convergence for all three limited-memory methods, too, which can be explained by the fact that all but one time point are either active or zero then.

Next we consider the convergence of $u$ and $q$ in different norms. Table 5 displays the following errors for the last four steps of each optimization method:

$$
e_{u, L^{2}}^{k}:=\left\|u^{k}-\bar{u}\right\|_{L^{2}}, \quad e_{u, H^{1}}^{k}:=\left\|u^{k}-\bar{u}\right\|_{H^{1}}, \quad e_{u, L^{\infty}}^{k}:=\left\|u^{k}-\bar{u}\right\|_{L^{\infty}},
$$

and analogue definitions $e_{q, L^{2}}^{k}, e_{q, H^{1}}^{k}, e_{q, L^{\infty}}^{k}$ for $q$. The semismooth Newton method exhibits one-step convergence as soon as the active sets have converged. The other methods show a quick decrease of all values towards the relative tolerance during 


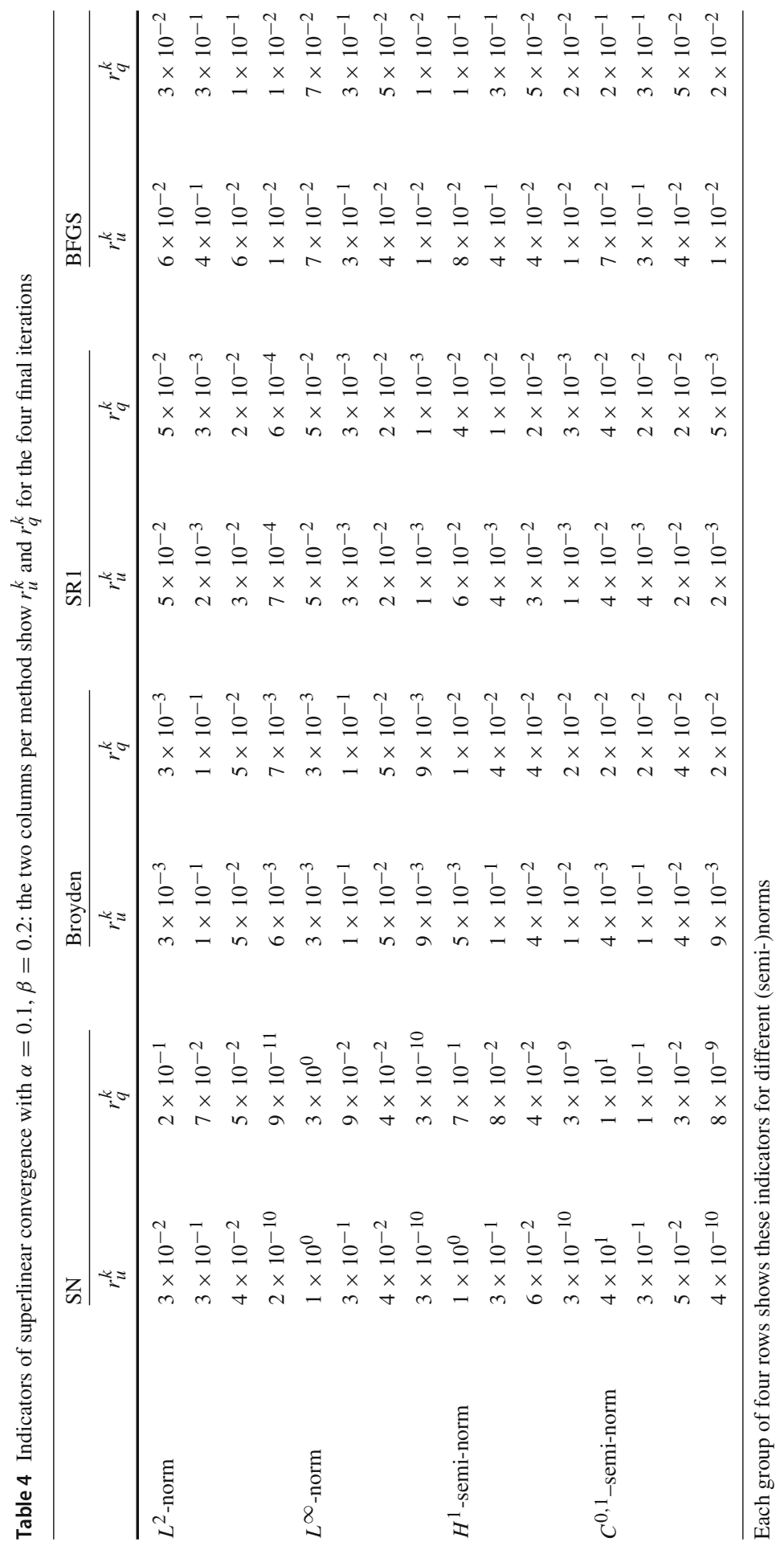


the last four steps of the optimization run. In particular, SR1 yields the fastest reduction, while BFGS shows a significantly slower convergence here. As predicted by Theorem 2, part (2), we have $e_{u, L^{2}}^{k} \leq e_{q, L^{2}}^{k}$ and $e_{u, L^{\infty}}^{k} \leq e_{q, L^{\infty}}^{k}$ (since these inequalities are a consequence of the fact that $\operatorname{Prox}_{\varphi_{1}}: L^{s} \rightarrow L^{s}$ is nonexpansive for any $s \in[1, \infty]$, they hold in all four methods under consideration). We observe that, in contrast, $e_{u, H^{1}}^{k} \leq e_{q, H^{1}}^{k}$ does not hold in general. However, the results indicate that $e_{u, H^{1}}^{k}$ still goes to zero, which agrees with part (2) of Theorem 2 for the choice $Q=H^{1}(I)$ described in Lemma 7 .

\subsection{Sparse control of the Bloch equation}

As example for a nonconvex optimization problem we investigate the bilinear control of the Bloch equations in magnetic resonance imaging (without relaxation, in the rotating frame, and on-resonance). A realistic optimal control modeling for radiofrequency (RF) pulse design in slice-selective imaging is considered based on Rund et al. (2018a). However, we add sparsity to the control model, which is a desirable feature in practice since the duty cycle of the RF amplifier is often limited. For details on magnetic resonance imaging we refer to Bernstein et al. (2004). As model problem we consider the slice-selective imaging with a single slice. Here, imaging data of a whole slice is to be acquired. The spatial field of view is described by its extent $\Omega \subset \mathbb{R}$ perpendicular to the slice direction. The slice itself is described by $\Omega_{\text {in }} \subset \Omega$ while the remaining part of $\Omega$ is denoted by $\Omega_{\text {out }}=\Omega \backslash \Omega_{\text {in }}$. The latter should not contribute to the data acquisition. The control problem is modeled as tracking of the nuclear magnetization vector $\mathbf{M}=\mathbf{M}(u)=\left(M_{1}, M_{2}, M_{3}\right)$ at the terminal time $T$. Specifically, we consider

$$
\begin{aligned}
& \min _{u \in U_{\mathrm{ad}}} \hat{f}(u)+\frac{\alpha}{2}\|u\|_{L^{2}(I)}^{2}+\beta\|u\|_{L^{1}(I)} \quad \text { s.t. } \\
& \dot{\mathbf{M}}(t, x)=\gamma \mathbf{M}(t, x) \times \mathbf{B}(t, x) \quad \text { a.e. in } I \times \Omega, \quad \mathbf{M}(0, x)=\mathbf{M}_{0}(x) \text { a.e. in } \Omega
\end{aligned}
$$

with $\alpha>0$, proton gyromagnetic ratio $\gamma=267.5380[\mathrm{rad} / \mathrm{s} / \mu \mathrm{T}]$, given initial condition $\mathbf{M}_{0}(x)$, spatial domain $x \in \Omega=(-c, c)$ with $c=0.06[\mathrm{~m}]$, and time $t \in I=(0, T)$ with $T=2.69[\mathrm{~ms}$ ]. The term $\hat{f}(u)$ is a tracking-type functional at the terminal time $T$ describing the intended use of the RF pulse, see below. The external magnetic field $\mathbf{B}(t, x)=(u(t), v(t), w(t) x)$ depends on the RF pulse $(u, v) \in L^{2}(I)^{2}$ and the slice-selective gradient amplitude $w=w(t) \in L^{2}(I)$. While these three timedependent functions can often be controlled, we consider for simplicity the situation in which $w \equiv 2$ is given and the RF pulses are real-valued, i.e., $v \equiv 0$. Hence, $u$ is the control variable. Technical limitations of the RF amplifier are modeled as control constraints $U_{\mathrm{ad}}=\left\{u \in L^{2}(I):|u| \leq u_{\max }\right\}$ with $u_{\max }=1.2\left[10^{2} \mu \mathrm{T}\right]$. This value reflects a typical $3 \mathrm{~T}$ magnetic resonance scanner hardware.

The specific example here is the optimization of a refocusing pulse, which is, among others, a central building block of the clinically important turbo spin echo based sequences. The initial condition results from assuming that an ideal $90^{\circ}$-excitation 


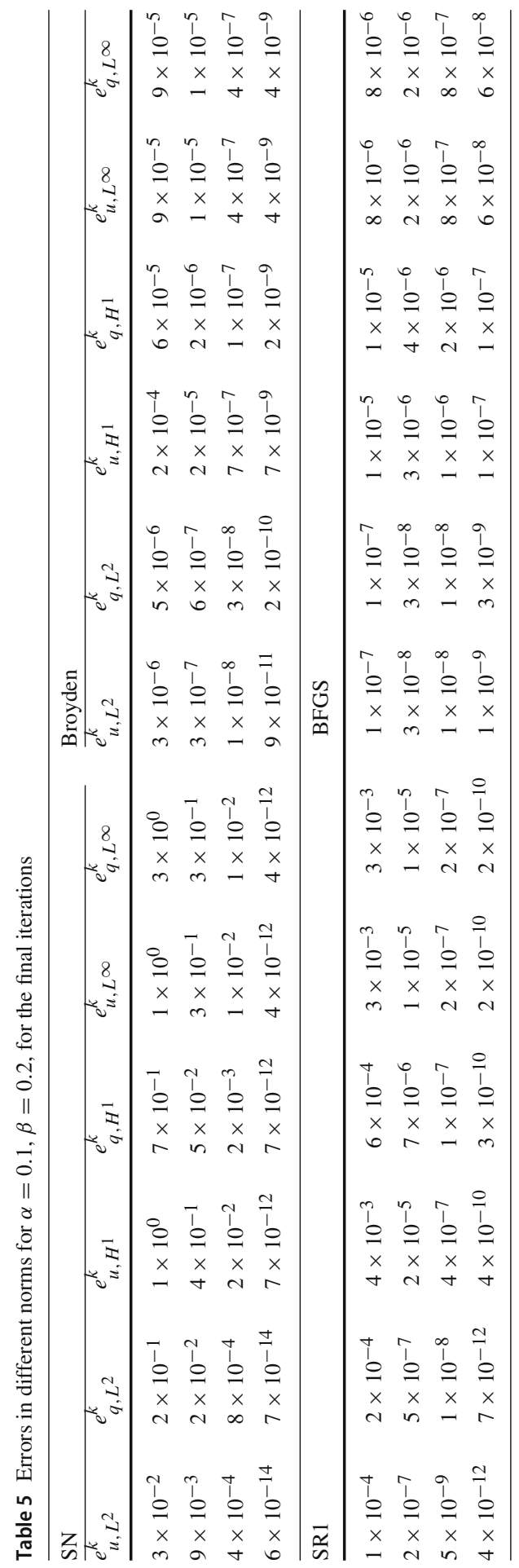


pulse for the same slice has been applied before, keeping the net magnetization vectors out of the slice in the steady state $(0,0,1)^{T}$ while exciting the slice itself. In particular, we set $\mathbf{M}_{0}=\chi_{\Omega_{\text {out }}}(x)(0,0,1)^{T}+\chi_{\Omega_{\text {in }}}(x)(0,1,0)^{T}$. A slice of $1.65[\mathrm{~cm}]$ thickness is assumed: $\Omega_{\text {in }}=[-0.00825,0.00825]$. The aim of the refocusing is to flip the magnetization vectors in the $x-y$-plane in the interior $\Omega_{\text {in }}$ of the slice, which is modeled as rotation around the $x$ axis with angle $\pi$. This desired magnetization pattern at the end time $t=T$ of the refocusing pulse is given by

$$
\hat{f}(u)=\frac{1}{2} \int_{\Omega_{\mathrm{in}}}\left(M_{1}(T, x)\right)^{2}+\left(M_{2}(T, x)+1\right)^{2} d x+\frac{1}{2} \int_{\Omega_{\mathrm{out}}}\left(1-M_{3}(T, x)\right)^{2} d x,
$$

recalling that $\mathbf{M}=\mathbf{M}(u)$. However, this tracking term for basic refocusing pulses is typically not used in numerical practice. Instead, we apply a more involved formulation of the desired state at the terminal time for advanced refocusing pulses, that we describe now. Because of practical reasons including robustness issues, refocusing pulses are generally applied within crusher gradients, cf. Bernstein et al. (2004), which are additional sequence elements surrounding the RF pulse. These crusher gradients cannot be modeled by the depicted $\hat{f}(u)$. It seems that the only practical way to model tracking terms for refocusing pulses with ideal crusher gradients is to define them in the spin domain, cf. Bernstein et al. (2004), Rund et al. (2018a). Therefore, we choose an equidistant time grid $t_{k}=(k-1) \tau, k=1, \ldots, N_{t}$ with $N_{t}=270$ points and step size $\tau=T /\left(N_{t}-1\right)=0.01[\mathrm{~ms}]$, together with piecewise constant $w$ and control $u$ with values $w_{m}, u_{m}, m=1, \ldots, N_{t}-1$. This implies that the magnetic field $\mathbf{B}$ is piecewise constant. It is well-known that for piecewise constant magnetic field the Bloch equations (14) in a spatial point $x_{0}$ can be solved analytically as a sequence of rotations. This is expressed by using the Cayley-Klein parameters $\left(a_{m}\right),\left(b_{m}\right) \in \mathbb{C}$, $m=1, \ldots, N_{t}-1$ with evolution

$$
a_{m}=\alpha_{m} a_{m-1}-\beta_{m}^{*} b_{m-1}, \quad b_{m}=\beta_{m} a_{m-1}+\alpha_{m}^{*} b_{m-1},
$$

and with initial conditions $a_{0}=1, b_{0}=0$, cf. Pauly et al. (1991). For the formula relating $a_{m}, b_{m}$ and $\mathbf{M}\left(t_{m-1}, x_{0}\right)$ see (Bernstein et al. 2004, eq.(2.15)). The coefficients $\alpha_{m}, \beta_{m}$ are given by

$$
\alpha_{m}=\cos \left(\phi_{m} / 2\right)+i \gamma \tau x_{0} w_{m} \sin \left(\phi_{m} / 2\right) / \phi_{m}, \quad \beta_{m}=i \gamma \tau u_{m} \sin \left(\phi_{m} / 2\right) / \phi_{m},
$$

with $\phi_{m}=-\gamma \tau \sqrt{u_{m}^{2}+\left(x_{0} w_{m}\right)^{2}}$. Since it is well-known that perfect refocusing with ideal crusher gradients is obtained through $|b(T, x)|^{2}=\chi_{\Omega_{\text {in }}}(x)$ for a.e. $x \in \Omega$, the tracking term is given by

$$
\hat{f}(u)=\frac{1}{2}\left\||b(T, x)|^{2}-\chi_{\Omega_{\text {in }}}(x)\right\|_{L^{2}(\Omega)}^{2} .
$$

Note that $b=b(u)$. In the numerical experiments we use $\hat{f}$ as defined in (15). The adjoint equation and the reduced gradient for this formulation are derived in the 
appendix of Rund et al. (2018a). The spatial domain is discretized equidistantly in $N_{x}=481$ points.

In accordance with the presentation in Sect. 4 we use a control-reduced problem formulation for (13-14). For the problem at hand this bears the advantage to iterate only on the small control vector with $N_{t}-1=269$ entries, but not on the large state vector with $3 N_{x} N_{t}=389610$ entries. Consequently, $B_{k}$ is a rather small matrix of format $269 \times 269$, and the linear algebra operations for its update and evaluation are cheap. In effect, the numerical effort is dominated clearly by the state and adjoint solves; concrete values are reported below.

We consider the same four optimization methods as in Sect. 6.1, i.e., a semismooth Newton method and the hybrid method with the Broyden, SR1 and BFGS update, respectively. If not mentioned otherwise, these methods are globalized with tr-cg. The stopping criterion is a relative tolerance of $10^{-5}$ for the residual norm $\|H\|_{L^{2}(I)}$. Unless declared otherwise, the following settings are applied for the hybrid methods: a limit of $L=75, B_{0}=0$ for Broyden and SR1 methods, and $B_{0}=\alpha I$ for BFGS. These initializations are selected because they turned out to be the most effective for the respective methods on this problem. Note that for Broyden and SR1 this is consistent with the numerical results for the optimal control of the heat equation in Sect. 6.1, cf. Table 1. Also, let us stress one more time that $B_{0}=0$ can be expected to yield a better performance than scaled identities, so we anticipate that Broyden and SR1 may be somewhat superior to BFGS in the following experiments whenever $B_{0}=0$, respectively, $B_{0}=\alpha I$ are used. This turns out to be true.

\subsubsection{Comparison of the optimization methods}

The optimization is initialized with a sinc-shaped RF pulse $f^{0}(t)=1.8 \cdot \operatorname{sinc}(-2.2+$ $4 t / T)$. To maintain a good initial slice profile we use $q^{0}=f^{0}+\operatorname{sign}\left(f^{0}\right) \beta / \alpha$, which implies $f^{0}=\sigma\left(q^{0}\right)$ and $u^{0}=\Pi_{U_{\text {ad }}}\left(\sigma\left(q^{0}\right)\right)=\Pi_{U_{\text {ad }}}\left(f^{0}\right)$. The initialization, the corresponding slice profile $M_{3}(T, x)$, and the desired slice profile are depicted in the top of Fig. 2. Also depicted are optimal controls for different $\alpha, \beta$. The sparsity and bound properties of the solutions for different $\alpha, \beta$ are depicted in Table 6 . If not mentioned otherwise, then we use $\alpha=5 \times 10^{-4}$ and $\beta=10^{-4}$ below. In all experiments we monitor that only runs leading to the same local minimizer are compared, which is important since the problem possesses several different minimizers.

The first study compares the performance of four different semismooth Newtontype methods embedded in a trust-region cg framework for varying $\alpha, \beta$ and for different initializations of the quasi-Newton matrix $B_{0}$. First, the limited-memory methods are analyzed in Table 7 and compared to the semismooth Newton method. The up to four columns per method show the iteration counts for different $B_{0}$. The last row shows the mean value per column taken over the converged runs only. The runs that do not converge are marked with - . The first three columns display the parameters $\alpha, \beta$ and the iteration number of SN. The next two column groups show that the hybrid method with Broyden updates behaves quite similar to the variant with SR1 updates, the latter often requiring slightly fewer iterations. The choices $B_{0}=0$ and $B_{0}=\alpha I$ yield fast convergence throughout all $(\alpha, \beta)$-pairings, while the other two formulas turn out to be less efficient in this setting. Looking at the hybrid method 


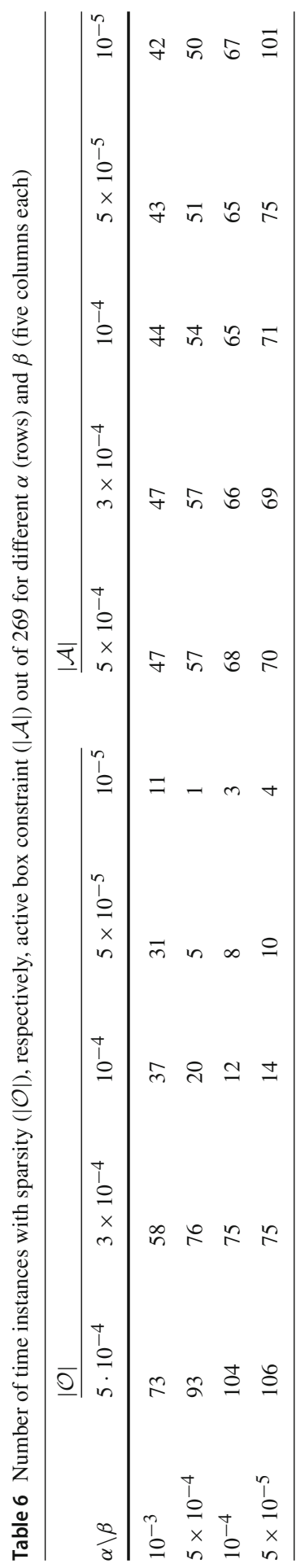



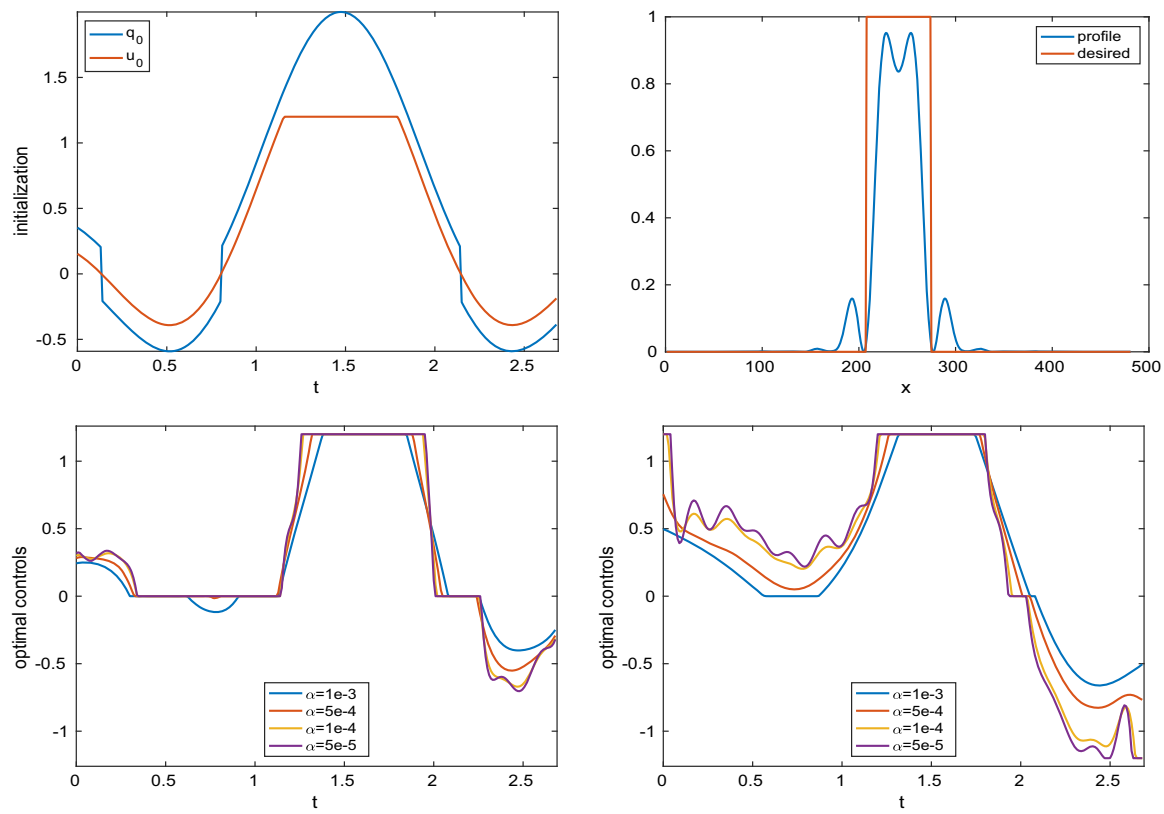

Fig. 2 Top: Initial $q^{0}$ and $u^{0}=\Pi_{U_{\mathrm{ad}}}\left(\sigma\left(q^{0}\right)\right)$ (left) and the corresponding slice profile $M_{3}(T, x)$ compared to the desired slice profile (right). Bottom: Optimal controls for different $\alpha$ with $\beta=5 \times 10^{-4}$ (left) and $\beta=5 \times 10^{-5}$ (right)

with BFGS in the last column group we observe that its performance degenerates for large values of $\alpha$. In view of the comment before Lemma 7 this is not entirely unexpected, but the extent of this behavior still appears somewhat surprising. Apart from this phenomenon the scaled identity yields good results also for BFGS. Using the best choice $B_{0}$ for each method, Broyden requires 41 iterations on average, SR1 35, and BFGS 83, compared to 16 for the semismooth Newton method. Since the latter has much more costly iterations due to the forward-backward solve of the second-order equations, it is important to compare the corresponding runtimes. They are included below, cf. Table 9 .

To address the choice of the limit parameter $L$ we compare the performance of the hybrid methods for different limits in Table 8 based on the iteration counts. Depicted are four columns per method which differ in the choice of the limit ranging from $L=25$ to $L=100$. The rows show results for different $\alpha$ while keeping $\beta=10^{-4}$ fixed. We stress that in combination with tr-cg it is appropriate to choose a limit $L$ that is larger than typical values from the literature for globalization by line search methods. This is due to the fact that Steihaug-cg employs earlier breaks in the cg method leading to smaller and more steps. We observe that a limit of 25 is only adequate for Broyden and SR1 in the case of large $\alpha \geq 5 \times 10^{-4}$. For smaller $\alpha$ the limit should be increased to 50. In contrast, the performance of BFGS is less sensitive to the values of $L$ that are investigated. 


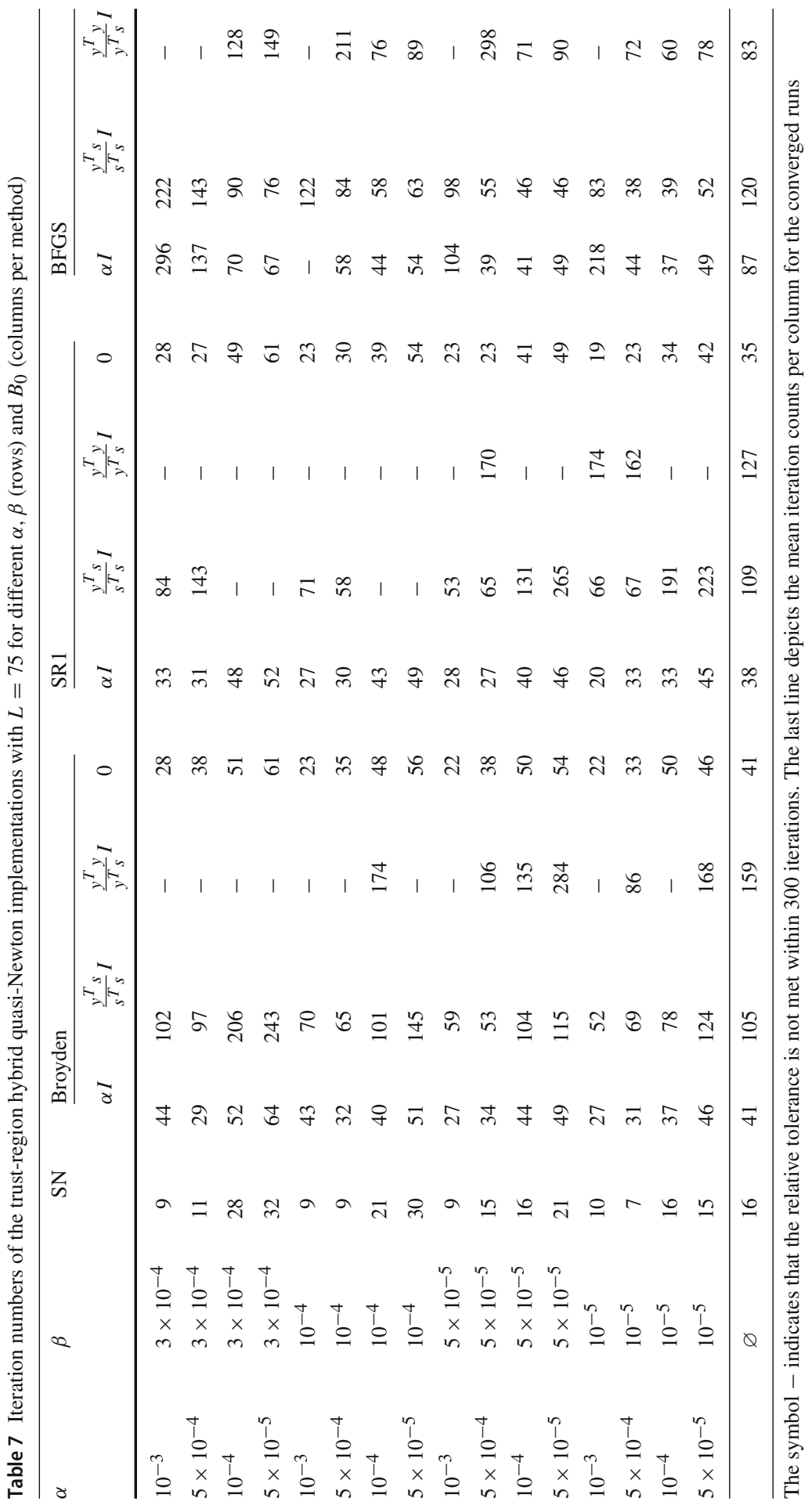


Table 8 Iterations of the hybrid quasi-Newton methods for different $\alpha$ and different limits $L$

\begin{tabular}{|c|c|c|c|c|c|c|c|c|c|c|c|c|}
\hline \multirow[b]{2}{*}{$\alpha \backslash L$} & \multicolumn{4}{|c|}{ Broyden } & \multicolumn{4}{|l|}{ SR1 } & \multicolumn{4}{|c|}{ BFGS } \\
\hline & 25 & 50 & 75 & 100 & 25 & 50 & 75 & 100 & 25 & 50 & 75 & 100 \\
\hline $10^{-3}$ & 23 & 23 & 23 & 23 & 23 & 23 & 23 & 23 & - & - & - & - \\
\hline $5 \times 10^{-4}$ & 61 & 35 & 35 & 35 & 69 & 30 & 30 & 30 & 54 & 58 & 58 & 58 \\
\hline $10^{-4}$ & 82 & 48 & 48 & 48 & - & 39 & 39 & 39 & 53 & 44 & 44 & 44 \\
\hline $5 \times 10^{-5}$ & 127 & 73 & 56 & 56 & 150 & 67 & 54 & 54 & 64 & 54 & 54 & 54 \\
\hline
\end{tabular}

The symbol - stands for not converging within 300 iterations

In the particular setting of optimal control problems with many state variables and few control variables, which is typically the case in practical applications of PDEconstrained optimization or Bloch-models, it pays off in runtime to use larger limits since this helps to save some iterations of the trust-region method while it increases the required time per trust-region iteration only marginally. This is due to the fact that the costs per trust-region iteration are largely dominated by the evaluation of objective and gradient. For example, the runtime of Broyden with $\alpha=5 \times 10^{-5}$ for a limit of 75 (56 iterations) is 5.1 seconds, which is significantly lower than the 6.4 seconds that are needed with a limit of 50 (73 iterations). In both cases, around $90 \%$ of the runtime is spent on the evaluation of objective and gradient. Therefore, a limit of $L=75$ is chosen for all subsequent studies. We emphasize that all runs converge to the same local minimizer independently of the limit parameter.

To investigate mesh independence properties of the hybrid methods we perform runs with different temporal and spatial mesh sizes. The results are shown in Table 9 using iteration counts in the left table, respectively runtime (mean runtime in 5 runs, in seconds) in the right table. The rows depict results for different temporal refinements with $N_{c}=N_{t}-1$ control points. The two columns per method show different spatial grids with $N_{x}=481$, respectively, $N_{x}=4811$ points. The finest example with $N_{c}=$ 17216 and $N_{x}=4811$ features 248 million degrees of freedom for the state variable. In all cases the same initial guess is used. Furthermore, the same local minimizer is attained in all runs, which allows for a direct comparison. The left table shows that the iteration counts of all four methods do not increase with $N_{c}$ or $N_{x}$. In particular, SN, Broyden and SR1 exhibit nearly the same iteration count for any of the discretizations. The hybrid method with BFGS displays a constant iteration number per column with a reduced iteration count for the right column (larger $N_{x}$ ). Interestingly, its performance is rather similar to that of Broyden and SR1 for $N_{x}=4811$, while for $N_{x}=481$ it requires roughly twice as many iterations and twice as much runtime as Broyden and SR1.

The right table shows the mean values of five runtimes in seconds, measured for a single CPU without parallelization. Despite their higher iteration counts, the three hybrid methods have much smaller runtimes than the semismooth Newton method. The bottom line depicts the mean value per column of the runtime in microseconds divided by $N_{x} N_{c}$, a number that varies only slightly per column (and per row, although not displayed). We regard this quantity as an efficiency index and denote it by $\mathcal{E}$. In 
contrast, the runtime of the semismooth Newton method increases faster in $N_{c}$ than linearly. We attribute this to the fact that the cg method requires more iterations for larger systems and that these iterations involve expensive operations for SN. Therefore, the speedup factor of the Broyden variant of the hybrid method over the semismooth Newton method rises with the number of time instances, starting at 7 and reaching 70 for $N_{c}=17216$ and $N_{x}=481$. Using SR1 updates leads to similar runtimes with a speedup of up to 68. As already seen in the left table, the use of BFGS updates produces higher iteration counts resulting in an increased runtime. Still, a speedup factor of up to 37 over the semismooth Newton method is reached.

Let us take a closer look at the convergence properties of the different quasi-Newton updates in the trust-region method. To this end, the solution is first computed in high precision with the semismooth Newton method and a relative tolerance of $10^{-13}$. Then the different optimization runs are performed with a relative tolerance of $10^{-7}$, measured in $\|H(\cdot)\|_{L^{2}(I)}$. The results are displayed in Table 10, with the error norms defined as in the first example, see (12). The table shows that all methods reduce all six errors to approximately the size of the relative tolerance, but the semismooth Newton method needs much fewer iterations to achieve this, cf. Table 9. We attribute this to the strong nonconvexity of the bilinear problem at hand. As in the first example we have $e_{u, L^{2}}^{k} \leq e_{q, L^{2}}^{k}$ and $e_{u, L^{\infty}}^{k} \leq e_{q, L^{\infty}}^{k}$, but this relationship is not satisfied for the $H^{1}(I)$-norm, which, however, does not impede $e_{u, H^{1}}^{k} \rightarrow 0$.

\subsubsection{Comparison of the globalization techniques}

This section is devoted to comparing the three globalizations tr-cg, ls-GMRES and nls-GMRES on the bilinear control problem of the Bloch equations. The globalizations are paired with the semismooth Newton method and the three limited-memory quasiNewton methods. For each of these twelve combinations, 2000 optimization runs from a random initial (MATLAB rand) $q^{0}$ are performed with the following parameters: $N_{c}=269, N_{x}=481, \alpha=5 \times 10^{-4}, \beta=10^{-4}$, up to 300 iterations, relative tolerance $10^{-4}, \mathrm{cg} / \mathrm{GMRES}$ tolerance $10^{-10}$, up to $100 \mathrm{cg} / \mathrm{GMRES}$ iterations. The monotone line search operates on the residual $\|H(q)\|_{L^{2}(I)}$, while the non-monotone line search is tested with $N_{\mathrm{ls}}=2,3,4,5$ based on the objective $f(u)+\varphi(u)$ and based on the residual $\|H(q)\|_{L^{2}(I)}$. Due to space limitations we show only the best results, which are obtained with $N_{\mathrm{ls}}=2$ and $R(u)=f(u)+\varphi(u)$.

Throughout the 8000 optimization runs with tr-cg, twelve different stationary points are observed, whose controls are depicted in Fig. 3, divided into three sets (top row). Every set of four controls yields identical optimal values $\bar{m}:=f(\bar{u})+\varphi(\bar{u})$, control norms $\|\bar{u}\|_{U}$, and final magnetization (bottom row). The four controls are related by axial symmetry to the $t$-axis and the axis $t=T / 2$. Since these four controls are equivalent in practical application, they are counted henceforth as one solution with multiplicity four. The relative occurrences of the resulting three candidates for a minimizer are depicted in the upper three rows of Table 11 in columns five to eight. The lower part of the table additionally displays the relative occurrences of runs that do not reach the prescribed relative tolerance within 300 iterations; they are labeled "not converged". The average objective value returned by the optimizer $\varnothing \bar{m}$ and 


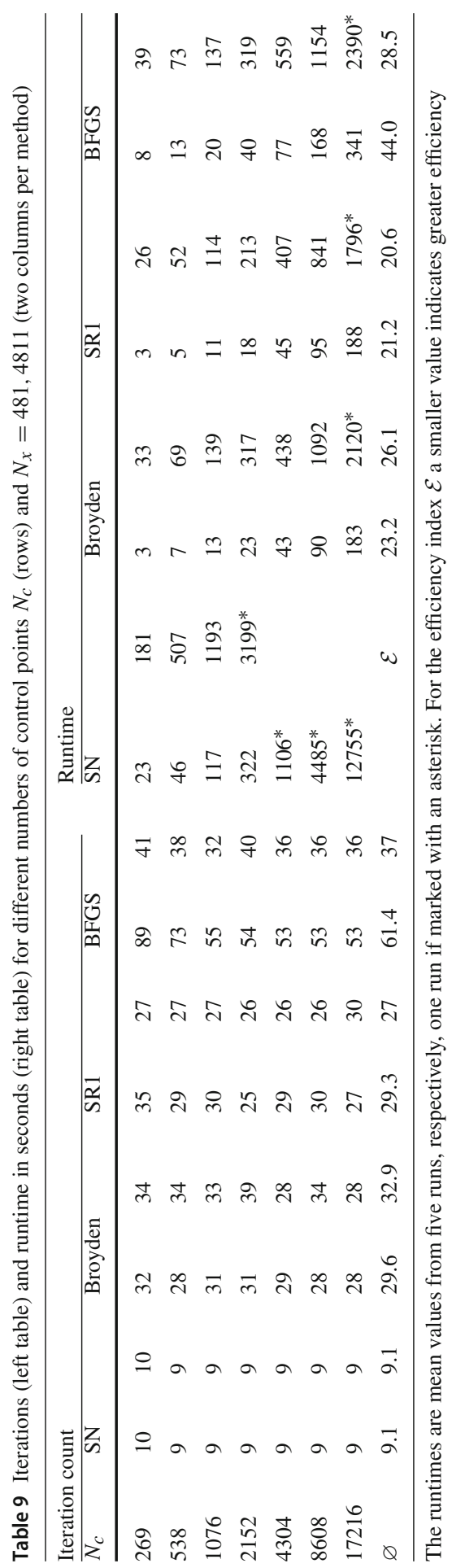




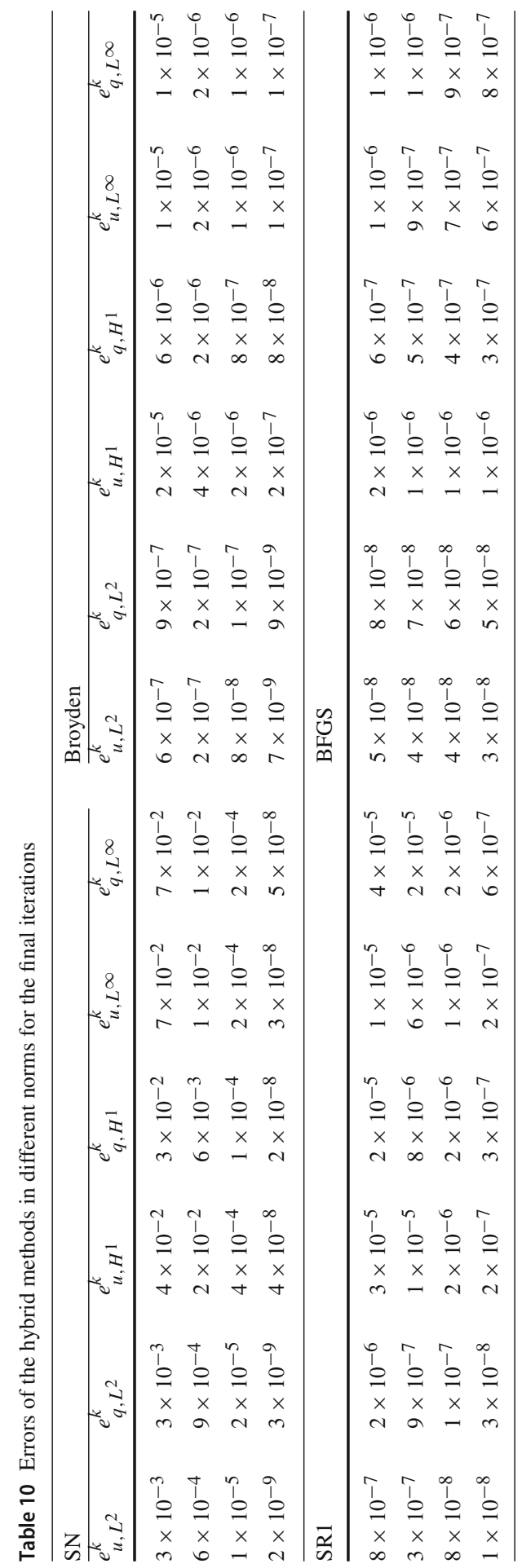



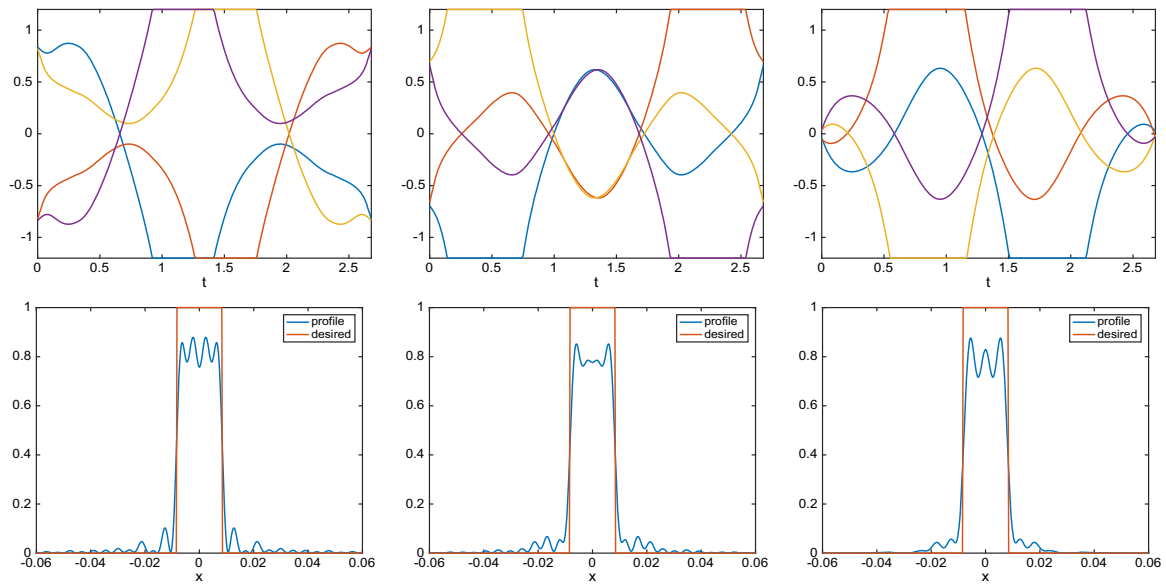

Fig. 3 Four optimized controls with the same objective value (top) and their slice profiles (bottom, desired state in red) for the three best observed candidates $\bar{m} \times 10^{3}=0.61906$ (left), $\bar{m} \times 10^{3}=0.64959$ (mid), and $\bar{m} \times 10^{3}=0.67280$ (right)

the average runtime in seconds are also shown. In contrast to previous experiments they are taken over all runs here, i.e., they include also the "not converged" runs. In particular, we observe that $\mathrm{SN}, \mathrm{Br}$ and $\mathrm{SR}$ always meet the relative tolerance. In contrast, one seventh of the BFGS runs does not converge. In fact, these runs yield the same twelve minimizers but fail to reach the prescribed tolerance, which is underlined by the agreeing values of $\varnothing \bar{m}$. This mean objective value is $\varnothing \bar{m}=0.64 \times 10^{-3}$ for all four methods with tr-cg, which is significantly smaller than the values achieved with the other globalization techniques. The mean runtime shows a clear speedup of the hybrid methods compared to $\mathrm{SN}$, despite the fact that this is a small scale example with $N_{t}=270$ and $N_{x}=481$.

In contrast to the trust-region method, the line search globalizations find many more stationary points, 78 in total. However, 49 of these have a prohibitively high cost. They are summarized in lines number 10 and 11 of Table 11 . The results with monotone line search (ls-GMRES) are depicted in the fourth column group of Table 11. The semismooth Newton method with a basic monotone line search on the residual $\|H(q)\|_{L^{2}(I)}$ quickly converges to a noncompetitive minimizer in nearly all cases. The three quasi-Newton methods yield smaller cost values in the mean, but most of the runs fail to match the prescribed relative tolerance. The mean optimal values of all four methods are much larger than those obtained with tr-cg.

The non-monotone line search nls-GMRES is more effective than ls-GMRES for all three quasi-Newton methods, see the last column group of Table 11. We note that the number of runs that do not converge is smaller than for the monotone line search. In particular, Broyden and BFGS converge in most of the cases. Moreover, the best control and the top three controls are found more often leading to much better average optimal values compared to ls-GMRES. However, excellent values similar to those of tr-cg are attained only for BFGS. Notably, the semismooth Newton method does not benefit from the non-monotone line search; it behaves similarly as with 1s-GMRES. It 


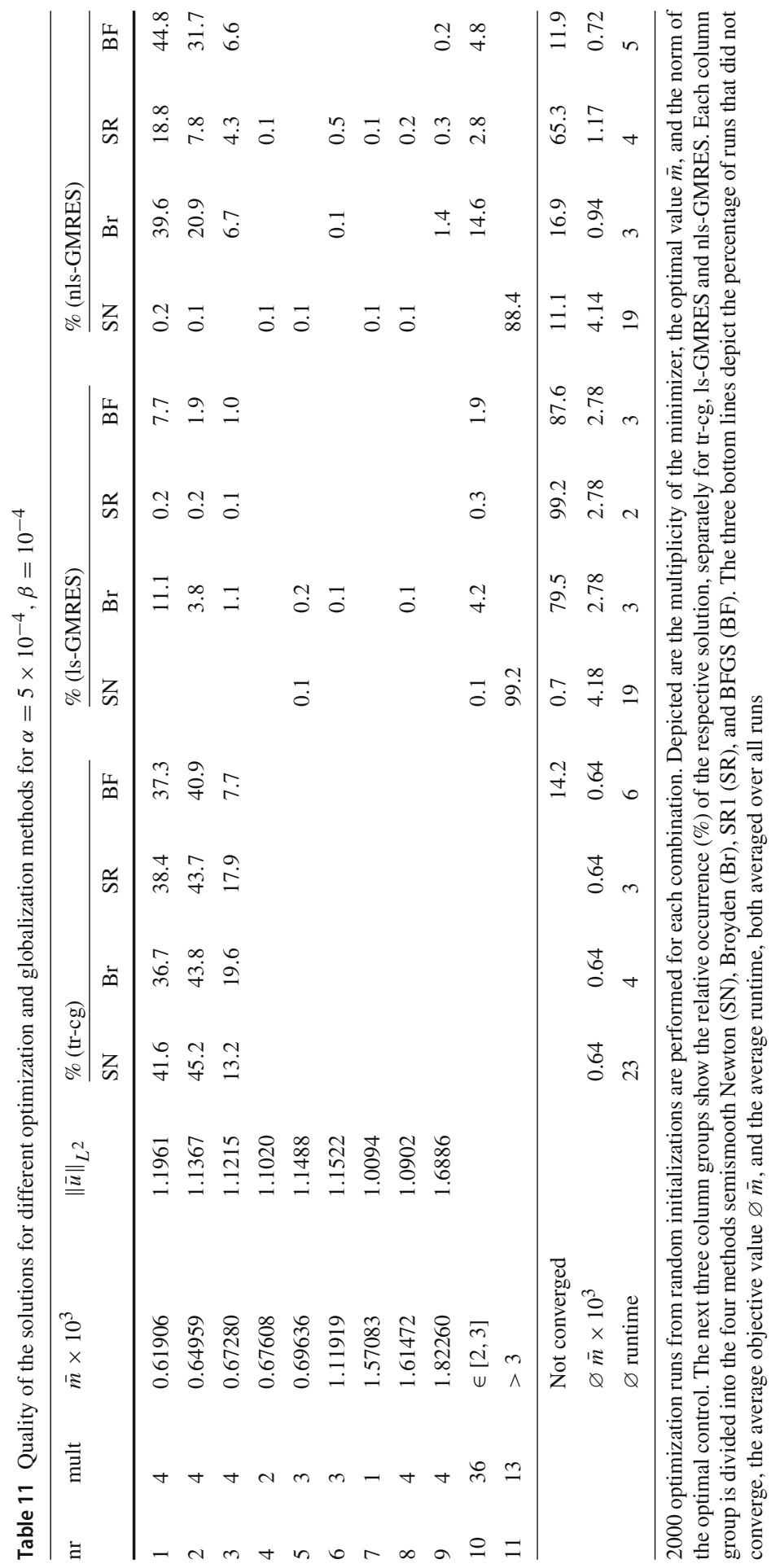


is also worth mentioning that the average runtimes of the tr-cg hybrid quasi-Newton methods are only slightly above those of the nls-GMRES variant.

Summarizing, in this application problem the tr-cg globalization robustly delivers the top-three candidates for all four optimization methods. In contrast, the line search globalizations often have difficulties with convergence for the quasi-Newton methods, and tend to noncompetitive solutions for the semismooth Newton method. Thus, for optimal control of the Bloch equations tr-cg should clearly be preferred over a line search globalization for the semismooth Newton, the hybrid Broyden, and the SR1 method. For BFGS, both tr-cg and nls-GMRES work equally well.

\section{Conclusions}

In this paper we have studied a hybrid approach for nonsmooth optimal control problems that blends semismooth Newton and quasi-Newton methods. We established its local superlinear convergence and provided numerical results to show that it has significantly lower runtime than semismooth Newton methods. A matrix-free limitedmemory truncated trust-region variant seems to be particularly promising.

Acknowledgements Open access funding provided by University of Graz. This work was supported in part by the Austrian Science Fund (FWF) in the context of the "SFB F32-N18" (Mathematical Optimization and Applications in Biomedical Sciences) in the Projects F3201 and F3202.

Open Access This article is licensed under a Creative Commons Attribution 4.0 International License, which permits use, sharing, adaptation, distribution and reproduction in any medium or format, as long as you give appropriate credit to the original author(s) and the source, provide a link to the Creative Commons licence, and indicate if changes were made. The images or other third party material in this article are included in the article's Creative Commons licence, unless indicated otherwise in a credit line to the material. If material is not included in the article's Creative Commons licence and your intended use is not permitted by statutory regulation or exceeds the permitted use, you will need to obtain permission directly from the copyright holder. To view a copy of this licence, visit http://creativecommons.org/licenses/by/4.0/.

\section{A Trust-region globalization of the hybrid method}

We state the precise algorithm of tr-cg that is employed in the numerical experiments. It is designed for solving (P) from Sect. 3 and uses the notation of that section. The objective of $(\mathrm{P})$ is denoted by $J: U \rightarrow \mathbb{R}$, i.e., $J(u):=f(u)+\varphi(u)$. For the norm $\|\cdot\|$ that appears in the algorithm we used $\|\cdot\|_{U}$, which worked well. The mapping $H$ is given by $H(q)=\nabla \hat{f}\left(\operatorname{Prox}_{\varphi_{\gamma}}(q)\right)+\gamma q$, cf. Lemma 1 . 


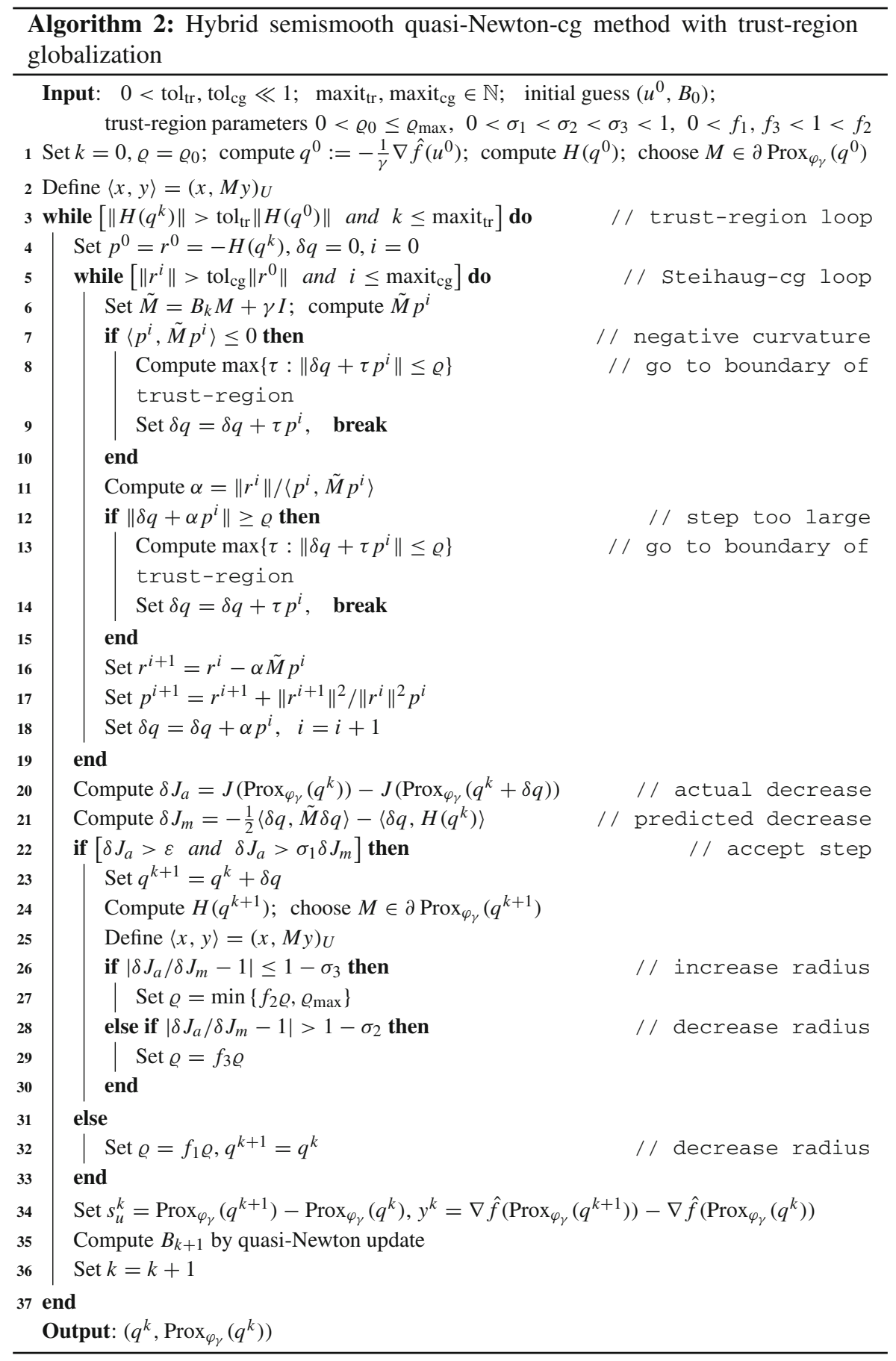




\section{References}

Adly S, Ngai HV (2018) Quasi-Newton methods for solving nonsmooth equations: generalized DennisMoré theorem and Broyden's update. J Convex Anal 25(4):1075-1104

Amstutz S, Laurain A (2013) A semismooth Newton method for a class of semilinear optimal control problems with box and volume constraints. Comput Optim Appl 56(2):369-403. https://doi.org/10. 1007/s10589-013-9555-6

Appell J, Zabrejko PP (1990) Nonlinear superposition operators. Cambridge tracts in mathematics. Cambridge University Press, Cambridge. https://doi.org/10.1017/CBO9780511897450

Bauschke HH, Combettes PL (2017) Convex analysis and monotone operator theory in Hilbert spaces, 2nd edn. Springer, Berlin. https://doi.org/10.1007/978-3-319-48311-5

Beck A (2017) First-order methods in optimization. MOS-SIAM series on optimization. SIAM, New Delhi. https://doi.org/10.1137/1.9781611974997

Bernstein MA, King KF, Zhou XJ (2004) Handbook of MRI pulse sequences. Elsevier Academic Press, Amsterdam. https://doi.org/10.1016/B978-012092861-3/50003-0

Borzì A, Schulz V (2012) Computational optimization of systems governed by partial differential equations, vol 8. SIAM, New Delhi. https://doi.org/10.1137/1.9781611972054

Boulanger AC, Trautmann P (2017) Sparse optimal control of the KdV-Burgers equation on a bounded domain. SIAM J Control Optim 55(6):3673-3706. https://doi.org/10.1137/15M1020745

Byrd RH, Nocedal J, Schnabel RB (1994) Representations of quasi-Newton matrices and their use in limited memory methods. Math Program 63(1 (B)):129-156. https://doi.org/10.1007/BF01582063

Casas E, Kruse F, Kunisch K (2017) Optimal control of semilinear parabolic equations by BV-functions. SIAM J Control Optim 55(3):1752-1788. https://doi.org/10.1137/16M1056511

Chen X, Yamamoto T (1992) On the convergence of some quasi-Newton methods for nonlinear equations with nondifferentiable operators. Computing 49(1):87-94. https://doi.org/10.1007/BF02238652

Chipot M (2000) Elements of nonlinear analysis. Birkhäuser, Basel. https://doi.org/10.1007/978-3-03488428-0

De los Reyes JC (2015) Numerical PDE-constrained optimization. Springer, Berlin. https://doi.org/10.1007/ 978-3-319-13395-9

Griewank A (1987) The local convergence of broyden-like methods on lipschitzian problems in hilbert spaces. SIAM J Numer Anal 24(3):684-705. https://doi.org/10.1137/0724045

Grissom WA, Setsompop K, Hurley SA, Tsao J, Velikina JV, Samsonov AA (2017) Advancing RF pulse design using an open-competition format: report from the 2015 ISMRM challenge. Magn Reson Med 78(4):1352-1361. https://doi.org/10.1002/mrm.26512

Han J, Sun D (1997) Newton and quasi-Newton methods for normal maps with polyhedral sets. J Optim Theory Appl 94(3):659-676. https://doi.org/10.1023/A:1022653001160

Herzog R, Stadler G, Wachsmuth G (2012) Directional sparsity in optimal control of partial differential equations. SIAM J Control Optim 50(2):943-963. https://doi.org/10.1137/100815037

Herzog R, Stadler G, Wachsmuth G (2015) Erratum: Directional sparsity in optimal control of partial differential equations. SIAM J Control Optim 53(4):2722-2723. https://doi.org/10.1137/15M102544X

Hinze M, Kunisch K (2001) Second order methods for optimal control of time-dependent fluid flow. SIAM J Control Optim 40(3):925-946. https://doi.org/10.1137/S0363012999361810

Hinze M, Pinnau R, Ulbrich M, Ulbrich S (2009) Optimization with PDE constraints, mathematical modelling: theory and applications, vol 23. Springer, Berlin. https://doi.org/10.1007/978-1-4020-88391

Ito K, Kunisch K (2008) Lagrange multiplier approach to variational problems and applications. SIAM, New Delhi. https://doi.org/10.1137/1.9780898718614

Kinderlehrer D, Stampacchia G (2000) An introduction to variational inequalities and their applications, reprint of the 1980 original edn. SIAM. https://doi.org/10.1137/1.9780898719451

Kunisch K, Rösch A (2002) Primal-dual active set strategy for a general class of constrained optimal control problems. SIAM J Optim 13(2):321-334

Kunisch K, Pieper K, Rund A (2016) Time optimal control for a reaction diffusion system arising in cardiac electrophysiology — a monolithic approach. ESAIM Math Model Numer Anal 50(2):381-414. https:// doi.org/10.1051/m2an/2015048

Mannel F, Rund A (2019) A hybrid semismooth quasi-Newton method for structured nonsmooth operator equations in Banach spaces. Submitted; preprint: https://imsc.uni-graz.at/mannel/sqn1.pdf 
Milzarek AM (2016) Numerical methods and second order theory for nonsmooth problems. PhD Thesis, Technische Universität München, Munich. https://nbn-resolving.de/urn/resolver.pl?urn:nbn:de:bvb: 91-diss-20160712-1289514-1-6

Muoi PQ, Hào DN, Maass P, Pidcock M (2013) Semismooth Newton and quasi-Newton methods in weighted $\ell^{1}$-regularization. J Inverse Ill-Posed Probl 21(5):665-693. https://doi.org/10.1515/jip-2013-0031

Nocedal J, Wright SJ (2006) Numerical optimization, 2nd edn. Springer series in operations research and financial engineering. Springer, Berlin. https://doi.org/10.1007/978-0-387-40065-5

Parikh N, Boyd SP (2014) Proximal algorithms. Found Trends Optim 1(3):127-239. https://doi.org/10. $1561 / 2400000003$

Pauly J, Le Roux P, Nishimura D, Macovski A (1991) Parameter relations for the Shinnar-Le Roux selective excitation pulse design algorithm. IEEE Trans Med Imaging 10(1):53-65. https://doi.org/10.1109/42. 75611

Pieper K (2015) Finite element discretization and efficient numerical solution of elliptic and parabolic sparse control problems. PhD Thesis, Technische Universität München, Munich. https://nbn-resolving.de/ urn/resolver.pl?nbn:de:bvb:91-diss-20150420-1241413-1-4

Qi L, Jiang H (1997) Semismooth Karush-Kuhn-Tucker equations and convergence analysis of Newton and quasi-Newton methods for solving these equations. Math Oper Res 22(2):301-325. https://doi. org/10.1287/moor.22.2.301

Robinson SM (1992) Normal maps induced by linear transformations. Math Oper Res 17(3):691-714. https://doi.org/10.1287/moor.17.3.691

Rund A, Aigner C, Kunisch K, Stollberger R (2018) Magnetic resonance RF pulse design by optimal control with physical constraints. IEEE Trans Med Imaging 37(2):461-472. https://doi.org/10.1109/ TMI.2017.2758391

Rund A, Aigner CS, Kunisch K, Stollberger R (2018) Simultaneous multislice refocusing via time optimal control. Magn Reson Med 80(4):1416-1428. https://doi.org/10.1002/mrm.27124

Sachs E (1985) Convergence rates of quasi-Newton algorithms for some nonsmooth optimization problems. SIAM J Control Optim 23:401-418. https://doi.org/10.1137/0323026

Schiela A (2008) A simplified approach to semismooth Newton methods in function space. SIAM J Optim 19(3):1417-1432. https://doi.org/10.1137/060674375

Stadler G (2009) Elliptic optimal control problems with $L^{1}$-control cost and applications for the placement of control devices. Comput Optim Appl 44(2):159-181. https://doi.org/10.1007/s10589-007-9150-9

Steihaug T (1983) The conjugate gradient method and trust regions in large scale optimization. SIAM J Numer Anal 20:626-637. https://doi.org/10.1137/0720042

Sun D, Han J (1997) Newton and quasi-Newton methods for a class of nonsmooth equations and related problems. SIAM J Optim 7(2):463-480. https://doi.org/10.1137/S1052623494274970

Tröltzsch F (2010) Optimal control of partial differential equations. Theory, methods and applications, vol 112. AMS, Providence. https://doi.org/10.1090/gsm/112

Ulbrich M (2011) Semismooth Newton methods for variational inequalities and constrained optimization problems in function spaces. MOS-SIAM series on optimization. SIAM, New Delhi. https://doi.org/ $10.1137 / 1.9781611970692$

Wang X, Ma C, Li M (2011) A globally and superlinearly convergent quasi-Newton method for general box constrained variational inequalities without smoothing approximation. J Glob Optim 50(4):675-694. https://doi.org/10.1007/s10898-010-9629-2

Xiao X, Li Y, Wen Z, Zhang L (2018) A regularized semi-smooth newton method with projection steps for composite convex programs. J Sci Comput 76(1):364-389. https://doi.org/10.1007/s10915-0170624-3

Publisher's Note Springer Nature remains neutral with regard to jurisdictional claims in published maps and institutional affiliations. 